\title{
ZONEAMENTO PALEOCLIMÁTICO DO QUATERNÁRIO DA BACIA DE SANTOS COM BASE EM FORAMINÍFEROS PLANCTÔNICOS
}

\author{
FABRICIO FERREIRA, ITAMAR IVO LEIPNITZ \\ Programa de Pós-Graduação em Geologia, Laboratório de Micropaleontologia, UNISINOS, Av. Unisinos, 950, 93022-000, \\ São Leopoldo, RS, Brasil.fabferreira@unisinos.br, itamar@unisinos.br \\ MARCO AURÉLIO VICALVI \& ANTONIO ENRIQUE SAYÃO SANJINÉS \\ CENPES/PDGEO/BPA, PETROBRAS, Av. Horácio Macedo, 950, 21941-915, Ilha do Fundão, Rio de Janeiro, RJ, Brasil. \\ vicalvi.gorceix@petrobras.com.br,antonio.sanjines@petrobras.com.br
}

\begin{abstract}
QUATERNARY PALEOCLIMATIC ZONATION OF SANTOS BASIN BASED ON PLANKTONIC FORAMINIFERA. The climatic zones based on planktonic foraminifera recovered from two piston cores collected on the slope of the Santos Basin, has shown the record of climate oscillations over the last $\sim 620 \mathrm{ka}$. Seven zones and 14 subzones were recognized, represented by glacial (zones $\mathrm{U}, \mathrm{W}$ and Y; subzones U2, U1, W2, W1, Y2, Y1B, Y1A) and interglacial intervals (zones T, V and X; subzones V3, V2B, V2A, V1, X1 to X3) of Pleistocene and the Holocene (zone Z). The Pulleniatina plexus allowed the division of subzone V2 in two (V2B and V2A) and provided eight regional correlation horizons along the subzones V3 and V2. The coiling direction of Globorotalia truncatulinoides helped the recognition of boundaries between subzones U2/U1, V2B/V2A, X3/X2 and X2/X1, showing as a useful tool for the refinement of the zones and subzones of the Quaternary. The permanent presence of suggests a constant influence of cold and productive waters over the past $\sim 620$ $\mathrm{ka}$, especially in the southern part of the study area.
\end{abstract}

Key words: biostratigraphy, planktonic foraminifera, Quaternary, correlation of biohorizons, paleoclimatic zoning.

RESUMO - O zoneamento climático, estabelecido a partir de foraminíferos planctônicos, para dois testemunhos coletados no talude da bacia de Santos, registrou as oscilações climáticas dos últimos $\sim 620$ ka. Foram reconhecidas sete zonas e 14 subzonas, representadas por intervalos glaciais (zonas U, W e Y; subzonas U2, U1, W2, W1, Y2, Y1B e Y1A) e interglaciais (zonas T, V e X; subzonas V3, V2B, V2A, V1, X1 a X3) do Pleistoceno e o Holoceno (Zona Z). O plexo Pulleniatina permitiu a divisão da subzona V2 em duas (V2B e V2A) e forneceu ao longo das subzonas V3 e V2 oito horizontes para a correlação regional. O controle do sentido de enrolamento de Globorotalia truncatulinoides auxiliou o reconhecimento dos limites entre as subzonas U2/U1, V2B/V2A, X3/X2 e X2/X1, demonstrando ser uma ferramenta útil para o refinamento das zona/ subzonas do Quaternário. A permanente presença de Globorotalia inflata sugere uma constante influência de águas frias e produtivas ao longo dos últimos $\sim 620 \mathrm{ka}$, em especial na região sul da área de estudo.

Palavras-chave: bioestratigrafia, foraminíferos planctônicos, Quaternário, biohorizontes de correlação, zoneamento paleoclimático.

\section{INTRODUÇÃO}

Os registros das oscilações climáticas que caracterizam o Quaternário (2,58 Ma; Gibbard et al., 2010) podem ser observados nos sedimentos marinhos a partir do controle da fauna de foraminíferos planctônicos. Ao longo deste período, a fauna de foraminíferos planctônicos sofreu flutuações significativas, devido a eventos migratórios com características não evolutivas ocorridos em resposta às variações do clima (Kennett \& Huddlestun, 1972; Bé et al., 1976; Thunnel, 1984). Desta forma, o controle da abundância relativa de assembleias e/ou espécies de foraminíferos planctônicos mais ou menos sensíveis às oscilações climáticas fornece uma ótima resolução estratigráfica para o Quaternário (Ericson \& Wollin, 1968; Thunell, 1984; Neff, 1985; Kohl et al., 2004).
As flutuações observadas ao longo do registro, como desaparecimento e reaparecimento de Globorotalia menardii em consequência das variações climáticas, foram originalmente reconhecidas no Atlântico Equatorial (Schott, 1935). Desde então, muitos trabalhos têm utilizado e validado esta metodologia (Phleger et al., 1953; Ericson \& Wollin, 1956; Ericson et al., 1961; Lidz, 1966), culminando na biozonação do Quaternário a partir da presença/ausência de G. menardii (Ericson \& Wollin, 1968), onde a presença indica intervalos climaticamente quentes (interglaciais) e a ausência intervalos climaticamente frios (glaciais). Enquanto o controle de algumas espécies e/ou associações reflete extremos climáticos, como o plexo G. menardii, outras são utilizadas por fornecer valiosas informações aos estudos bioestratigráficos do Quaternário, contribuindo assim com 
o aumento da resolução do zoneamento e, em consequência, melhorando a correlação entre seções.

Nas bacias brasileiras, o zoneamento baseado na presença/ ausência/abundância relativa de espécies e/ou associações chaves (como os plexos G. menardii e Pulleniatina) vem permitindo a correlação regional entre poços e auxiliado no estudo da evolução e dos eventos associados às bacias sedimentares. No entanto, até o momento, os estudos realizados com base em foraminíferos planctônicos abrangem apenas o intervalo mais recente do período, representado pelas zonas W, X, Y e Z (Vicalvi \& Palma, 1980; Rodrigues \& Carvalho, 1980; Vicalvi, 1997, 1999; Toledo, 2000; Portilho-Ramos et al., 2006; Sanjinés, 2006; Oliveira et al., 2007; Araújo \& Machado, 2008). Nos últimos anos, a bacia de Santos tem recebido considerável atenção da indústria do petróleo (Modica \& Brush, 2004; Duarte \& Viana, 2007), requerendo uma caracterização mais acurada de seus depósitos. Nesta perspectiva, este estudo tem como objetivo estabelecer o zoneamento paleoclimático dos depósitos quaternários da bacia de Santos com base na fauna de foraminíferos planctônicos. Paralelamente, pretende também caracterizar as zonas e subzonas bioestratigráficas identificadas.

\section{ÁREA DE ESTUDO}

A bacia de Santos é definida geologicamente como uma depressão localizada entre os paralelos $23^{\circ}-28^{\circ} \mathrm{S}$, ao longo da costa dos estados do Rio de Janeiro, São Paulo, Paraná e Santa Catarina, ocupando uma área aproximada de $3,5.10^{5} \mathrm{~km}^{2}$ (Figura 1). Ao norte, limita-se com a bacia de Campos no Alto de Cabo Frio, ao sul com a bacia de Pelotas na Plataforma de Florianópolis, a leste na cota batimétrica de $3.000 \mathrm{~m}$ e a oeste pela Serra do Mar, feição fisionômica que confina a bacia ao domínio marinho (Pereira \& Feijó, 1994; Moreira et al., 2007).

Os depósitos quaternários marinhos de águas profundas aqui abordados estão inseridos no contexto litoestratigráfico da Formação Marambaia, sequência N50-N60 (Moreira et al., 2007), onde predominam os sedimentos lamosos (folhelho cinzento e marga cinza-clara), cortados por cânions preenchidos por sedimentos lamosos e arenosos finos de origem turbidítica. Os sedimentos entre a plataforma interna e a externa apresentam características que variam de siliciclásticos a carbonáticos, respectivamente, enquanto o talude é caracterizado pela deposição de sedimentos hemipelágicos e turbiditos (Mahiques et al., 2002).

Segundo Viana et al. (1998), as massas de água superficiais atuantes na região são direcionadas em sentido sul por influência da Corrente do Brasil (CB) e compostas pela mistura de três massas de águas distintas, sendo elas a Água Costeira (AC; rasa, quente e com baixa salinidade), a Água Tropical (AT), quente e salina $\left(\mathrm{T}>18^{\circ} \mathrm{C} ; \mathrm{S}>36 \%\right.$ ), e a Água Central do Atlântico Sul (ACAS), mais fria e menos salina $\left(\mathrm{T}<18^{\circ} \mathrm{C} ; \mathrm{S}<36 \%\right.$ ). Ao sul da área de estudo, fluindo em sentido norte, encontra-se a Corrente das Malvinas (CM), caracterizada por águas frias e ricas em nutrientes (Wainer \& Venegas, 2002). O encontro das duas correntes ocorre entre $35^{\circ}-40^{\circ} \mathrm{S}$ e é denominado de Confluência Brasil-Malvinas (Brazil-Malvinas Confluence - BMC), caracterizando uma região de intensa produtividade (Boltovskoy et al., 1996). A intensidade das correntes superficiais (CB e CM) e o consequente deslocamento sazonal da BMC (norte-sul) são decorrentes, em geral, da variação do regime eólico e da migração da Zona de Convergência Intertropical (ZCIT) (Johns et al., 1998; Wainer \& Venegas, 2002). Desta forma, junto às descargas fluviais do Rio da Prata $\left(35^{\circ} \mathrm{S}\right)$ e da Lagoa dos Patos $\left(32^{\circ} \mathrm{S}\right)$, são importantes fontes de nutrientes atuantes no Atlântico Sul. Em condições adequadas, a pluma do Rio da Prata pode alcançar latitudes próximas a $23^{\circ} \mathrm{S}$, trazendo para a bacia de Santos uma elevada descarga de nutrientes (Campos et al., 1999).

A circulação profunda ocorre abaixo da ACAS, entre as cotas batimétricas de 600 e $3.500 \mathrm{~m}$, onde a Água Intermediária Antártica (AIA) que flui em sentido norte é caracterizada por temperaturas entre $2^{\circ}$ e $6^{\circ} \mathrm{C}$, salinidade mínima de 34,2\%o e alta concentração de oxigênio dissolvido. A Água Profunda do Atlântico Norte (APAN) flui em sentido sul, abaixo da AIA e apresenta temperatura entre $3^{\circ}$ e $4^{\circ} \mathrm{C}$ e salinidade entre 34,6 e 35\% (Silveira et al., 2000; Mahiques et al., 2004; Duarte \& Viana, 2007).

\section{MATERIAL E MÉTODOS}

Para esse estudo foram utilizados os testemunhos de sondagem BS-C e BS-D (Figura 1) e suas descrições sedimentológicas, cedidos pela PETROBRAS (Petróleo Brasileiro S.A.). Ambos provêm do talude da bacia de Santos e foram coletados no ano de 2007, sob lâmina de água maior que $2.100 \mathrm{~m}$, apresentando recuperação contínua superior a $20 \mathrm{~m}$ (Tabela 1).

Para as análises micropaleontológicas foram retiradas 188 amostras (BS-C: 108; BS-D: 80) com auxílio de um "plug" de PVC com volume interno de $10 \mathrm{~cm}^{3}$, em intervalos regulares de $30 \mathrm{~cm}$ e/ou quando houvesse alteração litológica. Em laboratório, as amostras foram tratadas a partir da metodologia padrão para a preparação de microfósseis calcários (Leipnitz et al., 2005). Para a construção do arcabouço bioestratigráfico, optou-se pela utilização da fração $>125 \mu \mathrm{m}$, por concentrar os foraminíferos planctônicos de maior interesse para a subdivisão do Quaternário (Kennett \& Huddlestun, 1972; Bé et al., 1976; Martin et al., 1990). Esta fração foi quarteada quando necessário, para a obtenção de um número

Tabela 1. Localização geográfica, profundidade de coleta (lâmina d'água), recuperação $(\mathrm{m})$ e número de amostras coletadas nos testemunhos BS-C e BS-D.

Table 1. Geographical location of cores BS-C and BS-D, water depth $(\mathrm{m})$, recovery $(\mathrm{m})$ and number of samples collected.

\begin{tabular}{ccccc}
\hline Testemunho & $\begin{array}{c}\text { Coordenadas } \\
\text { geográficas }\end{array}$ & $\begin{array}{c}\text { Lâmina } \\
\text { d'água }(\mathrm{m})\end{array}$ & $\begin{array}{c}\text { Recuperação } \\
(\mathrm{m})\end{array}$ & $\begin{array}{c}\text { Número de } \\
\text { amostras }\end{array}$ \\
\hline \multirow{2}{*}{ BS-C } & $\begin{array}{c}25^{\circ} 51.5^{\prime} \mathrm{S} \\
43^{\circ} 33.8^{\prime} \mathrm{W}\end{array}$ & 2.148 & 20,43 & 108 \\
& $\begin{array}{c}25^{\circ} 57.9^{\prime} \mathrm{S} \\
\text { BS-D }\end{array}$ & $\begin{array}{c}2.171 \\
3^{\circ} 24.1^{\prime} \mathrm{W}\end{array}$ & 21,21 & 80 \\
\hline
\end{tabular}




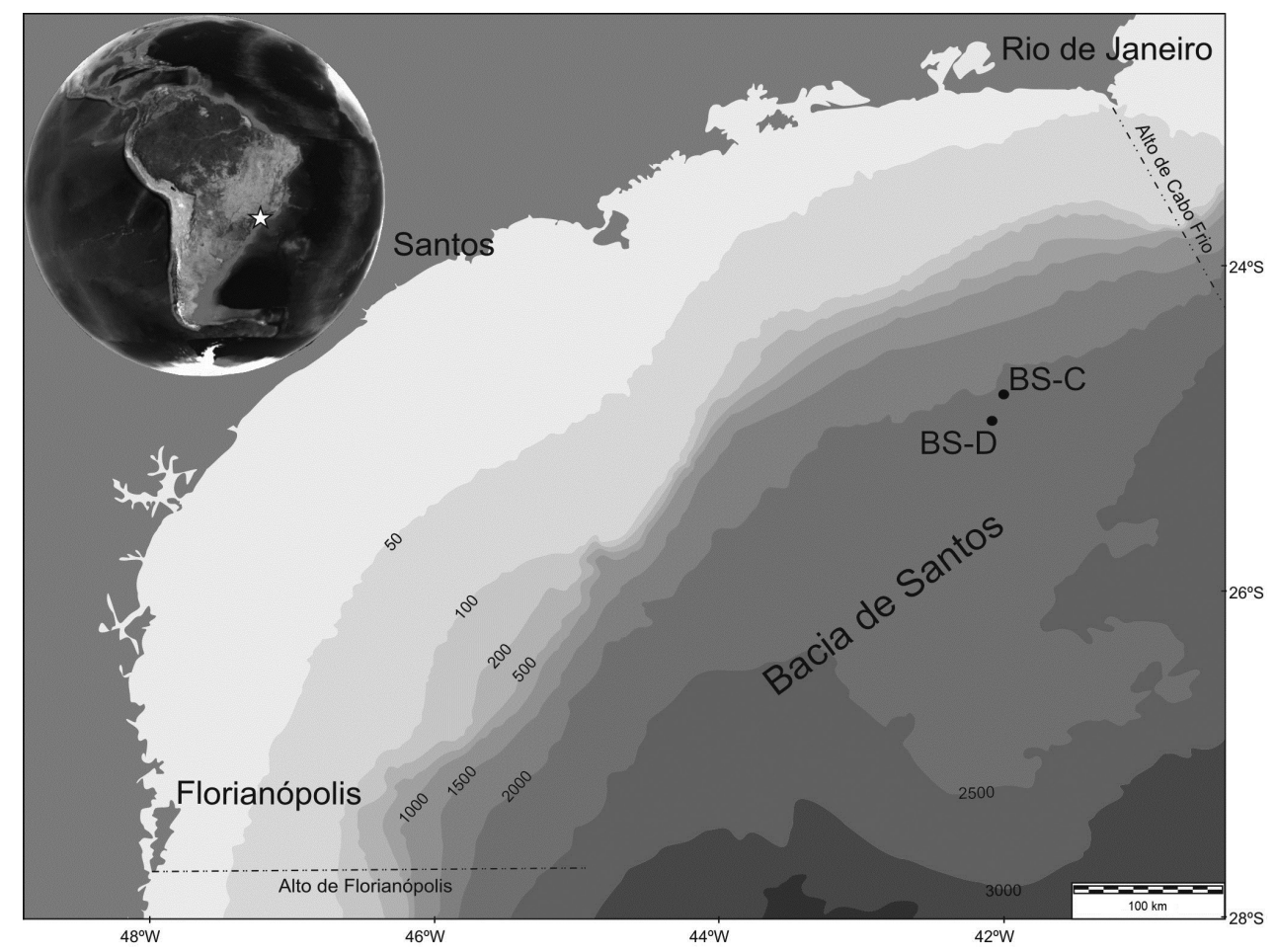

Figura 1. Mapa de localização da bacia de Santos e dos testemunhos BS-C e BS-D (modificado de Nunes et al., 2004).

Figure 1. Location map of Santos Basin and piston cores BS-C and BS-D (modified from Nunes et al., 2004).

significativo de foraminíferos planctônicos e bentônicos (entre 300 e 600, segundo Patterson \& Fishbein, 1989).

É importante ressaltar que, neste trabalho, as zonas/ subzonas identificadas refletem as mudanças ocorridas na composição das associações e/ou espécies de foraminíferos planctônicos diagnósticas para a bioestratigrafia do Quaternário. As espécies foram selecionadas de acordo com sua tolerância às variações ambientais, principalmente em relação à temperatura, salinidade, produtividade e alterações na estrutura vertical da coluna d'água (e.g. alterações na profundidade da termoclina e/ou nutriclina). O plexo Globorotalia menardii, composto pelas espécies G. menardii menardii, G. menardii flexuosa, G. tumida e G. fimbriata, é o principal representante dos intervalos interglaciais (águas quentes: tropicais e subtropicais), tendo sua distribuição controlada principalmente pela temperatura (Kennett \& Huddleston, 1972; Rögl \& Bolli, 1973; Bé et al., 1976; Prell \& Damuth, 1978; Martin et al., 1990, 1993; Boltovskoy et al., 1996; Martinez et al., 2007). O plexo Pulleniatina, composto pelas espécies $P$. obliquiloculata, $P$. primalis e $P$. finalis, geralmente habita regiões tropicais e subtropicais. Mais tolerante ao frio que o plexo G. menardii, a distribuição do plexo Pulleniatina parece ser controlada principalmente pela temperatura e salinidade, no entanto, alguns autores sugerem que a produtividade seja um importante fator a ser considerado (Bé \& Tolderlund, 1971; Cifelli \& Berniér, 1976; Prell \& Damuth, 1978; Boltovskoy et al., 1996; PortilhoRamos, 2010).

A espécie Globorotalia inflata demonstra preferência por águas transicionais a frias, habitando regiões de transição entre massas d'águas subtropicais e subpolares e zonas de ressurgência (Boltovskoy et al., 1996; Chiessi et al., 2007). A espécie Globorotalia truncatulinoides habita regiões temperadas a subpolares e a direção de seu enrolamento tem sido utilizada com sucesso em interpretações bioestratigráficas e paleoceanográficas. As variações na abundância de $G$. truncatulinoides (dex) vêm sendo utilizadas para avaliar alterações na profundidade da camada de mistura (Martin et al, 1990; Lohmann \& Schweitzer, 1990; Toledo, 2000; Schmidt et al., 2004; Toledo et al., 2007). Estas espécies ( $G$. inflata e $G$. truncatulinoides) são consideradas indicadoras de águas frias para o Atlântico Sul (Bolstovskoy, 1968, 1973; Bé \& Tolderlund, 1971; Bé et al., 1976). As demais espécies (Globorotalia crassaformis, Neogloboquadrina dutertrei, Globigerinoides conglobatus e Orbulina universa) foram selecionadas por apresentarem registro contínuo ao longo dos testemunhos e predomínio em determinados intervalos, auxiliando o zoneamento e a correlação entre seções (Kennett \& Huddlestun, 1972; Vicalvi, 1999). Representantes de cada espécie utilizada para o zoneamento bioestratigráfico foram depositados no Museu da História da Vida e da Terra (MHGeo - UNISINOS) sob a numeração ULVG-8601 a ULVG-8612 (Figura 2).

\section{Horizontes de correlação e arcabouço biocronoestratigráfico}

Foram adotadas as idades locais propostas por Vicalvi $(1997,1999)$ e as idades sugeridas por Damuth (1975), Martin et al. (1993), Martin \& Fletcher (1995) e Kohl et al. (2004) para os limites entre zonas/subzonas (Tabela 2). O zoneamento climático estabelecido para a área inclui as zonas de Blow (1969), Bolli \& Premoli Silva (1973) e o zoneamento climático de Ericson \& Wollin (1968), primeira e última ocorrência de espécies, horizontes de correlação (Tabela 3) e a média da 
abundância relativa das espécies selecionadas. Para a correlação entre os testemunhos utilizou-se a curva do plexo G. menardii e as interpretações bioestratigráficas de cada testemunho. Estes dados foram confrontados com uma escala cronoestratigráfica, com as idades absolutas descritas na literatura e calculados a partir da estimativa da taxa de acumulação para os limites entre subzonas (Tabela 2) e horizontes de correlação locais reconhecidos (Tabela 3 ).

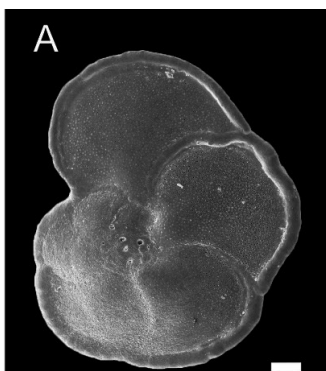

B

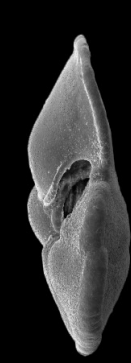

G

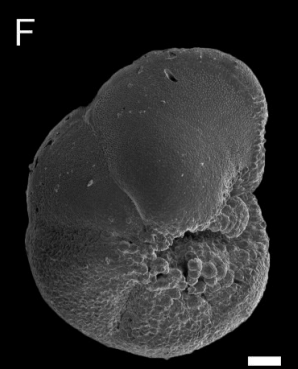

K

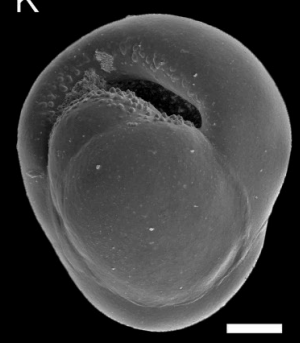

P
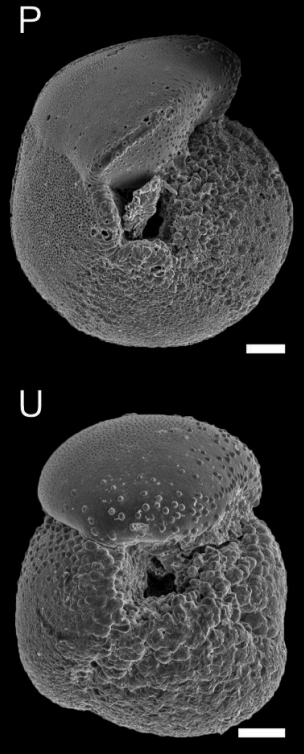

L

$Q$

V

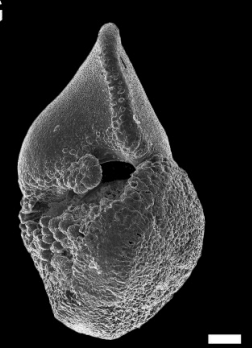

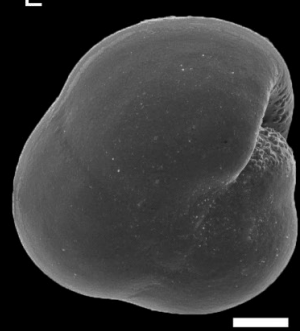
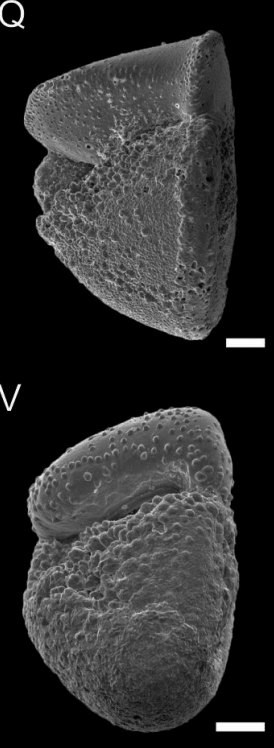

C

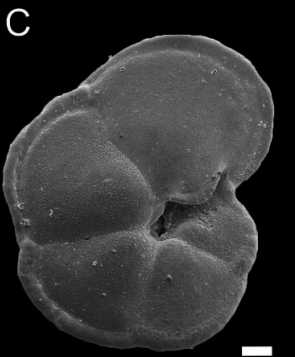

$\mathrm{H}$

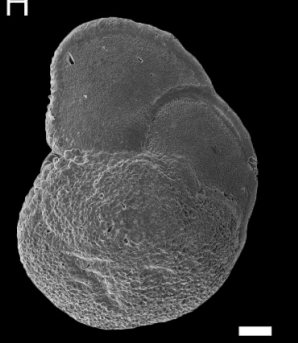

D

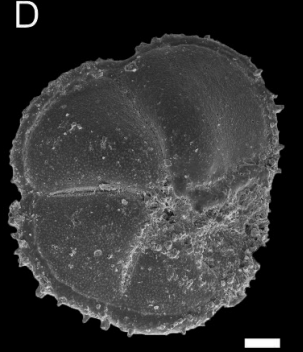

I

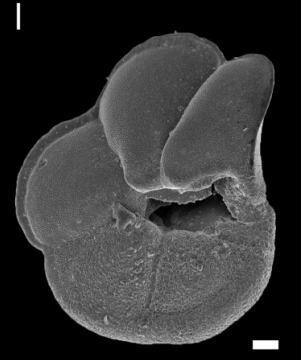

M

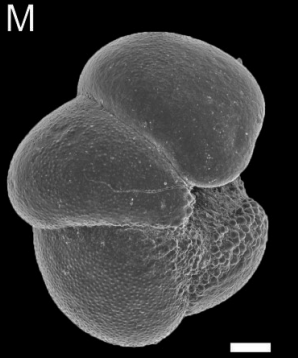

R

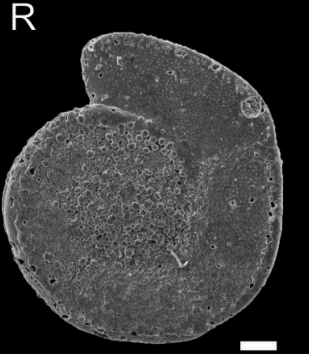

W

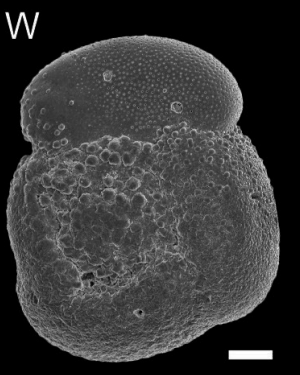

$N$

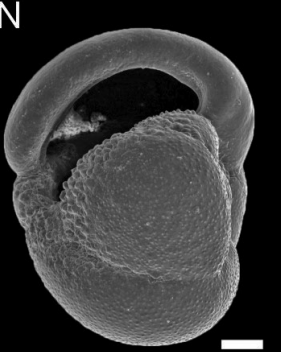

S

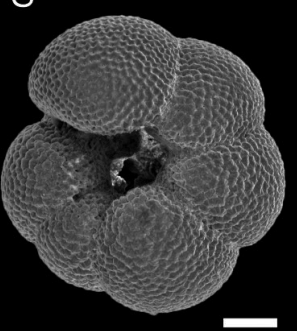

X

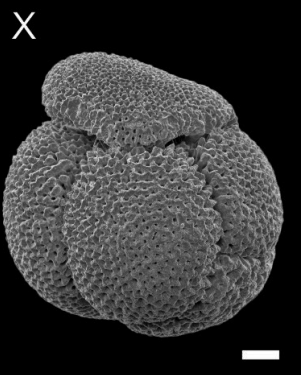

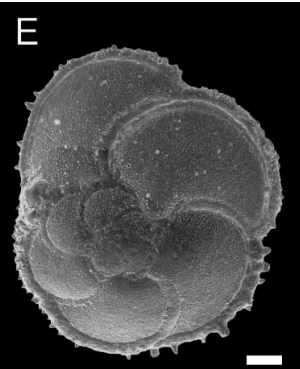

J

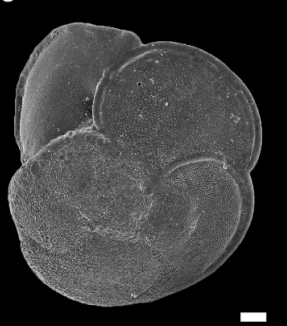

0

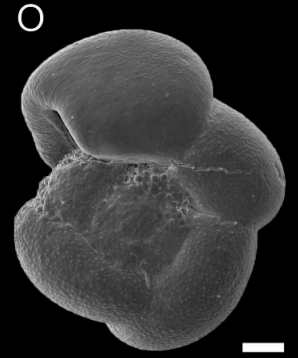

$\mathrm{T}$

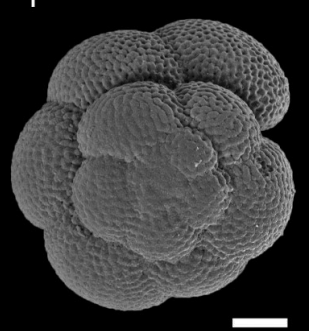

Y

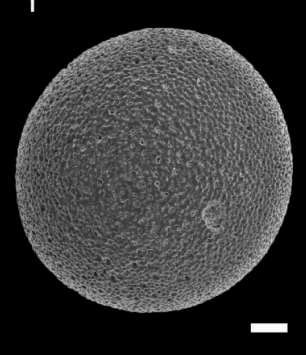

Figura 2. Espécies de foraminíferos planctônicos utilizadas para o zoneamento bioestratigráfico do Quaternário da bacia de Santos. A-C, Globorotalia menardii menardii; D-E, Globorotalia fimbriata; F-H, Globorotalia tumida; I-J, Globorotalia menardii flexuosa; K-L, Pulleniatina obliquiloculata; M-O, Globorotalia inflata; P-R, Globorotalia truncatulinoides; S-T, Neogloboquadrina dutertrei; U-W, Globorotalia crassaformis; $\mathbf{X}$, Globigerinoides conglobatus; Y, Orbulina universa. Escalas $=100 \mu \mathrm{m}$.

Figure 2. Species of planktonic foraminifera used for biostratigraphic zonation of the Quaternary of Santos Basin. Scale bars $=100 \mu \mathrm{m}$. 
Tabela 2. Limites entre zonas e subzonas nos testemunhos BS-C e BS-D, idades adotadas e referências utilizadas. Abreviaturas: (a) Broecker et al. (1960); (b) Bé et al. (1976); Vicalvi (1999); Sanjinés (2006); (c) Prell \& Damuth (1978); Vicalvi (1997, 1999); Sanjinés (2006); (d) Broecker et al. (1960); Damuth (1975); (e) Idade calculada com base na taxa de acumulação estimada; (f) Kohl et al. (2004); (g) Kennett \& Huddlestun (1972); Vicalvi (1997, 1999); Sanjinés (2006); (h) Novas subzonas (este trabalho); (i) Martin \& Fletcher (1995); (j) Neff (1985); Martin et al. (1990, 1993).

Table 2. Zone and subzone boundaries in the cores BS-C and BS-D, ages adopted and references used.

\begin{tabular}{|c|c|c|c|c|c|c|}
\hline & \multirow[t]{2}{*}{ Limites entre zonas e subzonas } & \multirow[t]{2}{*}{ Idade (ka) } & \multicolumn{2}{|c|}{ Idade estimada (ka) } & \multicolumn{2}{|c|}{ Profundidade do testemunho $(\mathrm{m})$} \\
\hline & & & BS-C & BS-D & BS-C & BS-D \\
\hline (a) & $\mathrm{Y} / \mathrm{Z}$ & 11 & & & 0,25 & 0,22 \\
\hline (b) & Y1B/Y1A & 15 & & & 0,55 & 0,58 \\
\hline (c) & $\mathrm{Y} 2 / \mathrm{Y} 1 \mathrm{~B}$ & $42 / 45$ & & & 0,90 & 1,25 \\
\hline (d) & $\mathrm{X} / \mathrm{Y}$ & 84 & & & 2,37 & 4,25 \\
\hline (e) & $\mathrm{X} 2 / \mathrm{X} 1$ & & 93,81 & 99,74 & 2,78 & 4,85 \\
\hline (e) & $\mathrm{X} 3 / \mathrm{X} 2$ & & 118,08 & 115,79 & 3,80 & 5,46 \\
\hline (f) & $\mathrm{W} / \mathrm{X}$ & 130 & & & 4,30 & 6,00 \\
\hline (e), (g) & $\mathrm{W} 2 / \mathrm{W} 1$ & & 162,35 & 148,52 & 4,74 & 6,45 \\
\hline (f) & $\mathrm{V} / \mathrm{W}$ & 186 & & & 5,06 & 7,35 \\
\hline (e) & $\mathrm{V} 2 \mathrm{~A} / \mathrm{V} 1$ & & 270,84 & 295,44 & 8,25 & 11,65 \\
\hline (e), (h) & $\mathrm{V} 2 \mathrm{~B} / \mathrm{V} 2 \mathrm{~A}$ & & 357,27 & 379,11 & 11,50 & 14,93 \\
\hline (e), (i) & V3/V2B & 400 & 399,83 & 400,07 & 13,10 & 15,75 \\
\hline (f) & $\mathrm{U} / \mathrm{V}$ & 485 & & & 16,32 & 19,35 \\
\hline (e), (j) & $\mathrm{U} 2 / \mathrm{U} 1$ & & 501,64 & 499,78 & 16,88 & 19,94 \\
\hline (f) & $\mathrm{T} / \mathrm{U}$ & 610 & & & 19,84 & - \\
\hline
\end{tabular}

Tabela 3. Data globais e locais identificados nos testemunhos BS-C e BS-D, profundidade ( $m$ ), idades (ka), limites entre zonas e subzonas e referências utilizadas. Abreviaturas: (a) Datum Primeiro Aparecimento (DPA) - Bolli \& Premolli Silva (1973); (b) Datum Último Reaparecimento (DUR) - Bé et al. (1976); Vicalvi (1999); (c) Datum Último Desaparecimento (DUD) - Prell \& Damuth (1975); Vicalvi (1997, 1999); PortilhoRamos et a. (2006); (d) Datum Penúltimo Desaparecimento (DPD) - Kennett \& Huddlestun (1972); Vicalvi (1997, 1999); (e) Última ocorrência Local (UOL) - Kennett \& Huddlestun (1972); Damuth (1975); Vicalvi (1997, 1999); (f) Datum Reaparecimento Local (DRL) - Novo Datum (este trabalho); (g) Datum Desaparecimento Local (DDL) - Novo Datum (este trabalho); * Idade Estimada com base na taxa de acumulação; ** Novas subzonas (este trabalho).

Table 3. Global and local data identified on cores BS-C and BS-D, depth $(\mathrm{m})$, ages (ka), zones and subzones boundaries and references used.

\begin{tabular}{|c|c|c|c|c|c|c|}
\hline & \multirow[t]{2}{*}{ Data locais e globais } & \multicolumn{2}{|c|}{ Testemunho (m) } & \multicolumn{2}{|c|}{ Idade (ka) } & \multirow[t]{2}{*}{ Limites } \\
\hline & & BS-C & BS-D & BS-C & BS-D & \\
\hline (a) & DPA Globorotalia fimbriata & & 0,22 & \multicolumn{2}{|c|}{11} & $\mathrm{Y} / \mathrm{Z}$ \\
\hline (b) & DUR plexo Pulleniatina (YP.4) & 0,55 & 0,58 & \multicolumn{2}{|c|}{15} & $\mathrm{Y} 1 \mathrm{~B} / \mathrm{Y} 1 \mathrm{~A}$ \\
\hline (c) & DUD plexo Pulleniatina (YP.3) & 0,90 & 1,25 & \multicolumn{2}{|c|}{$45-45$} & Y2/Y1B \\
\hline (d) & DPD plexo Pulleniatina (YP.2) & 2,02 & 3,20 & \multicolumn{2}{|c|}{$67,7-74,4$} & \\
\hline (e) & UOL G. menardii flexuosa & 2,37 & 4,25 & \multicolumn{2}{|c|}{84} & $\mathrm{X} 1 / \mathrm{Y} 2$ \\
\hline (f) & DRL plexo Pulleniatina (VP.8) & 11,50 & 14,93 & $357,27^{*}$ & $379,11^{*}$ & $\mathrm{~V} 2 \mathrm{~B} / \mathrm{V} 2 \mathrm{~A} * *$ \\
\hline (g) & DDL plexo Pulleniatina (VP.7) & 13,10 & 15,75 & $399,83^{*}$ & $400,03 *$ & V3/V2B \\
\hline (f) & DRL plexo Pulleniatina (VP.6) & 13,50 & 16,05 & $410,55^{*}$ & $407,07^{*}$ & \\
\hline (g) & DDL plexo Pulleniatina (VP.5) & 13,81 & 16,95 & $416,74^{*}$ & $428,30^{*}$ & \\
\hline (f) & DRL plexo Pulleniatina (VP.4) & 14,85 & 17,91 & $446,17^{*}$ & $450,94 *$ & \\
\hline (g) & DDL plexo Pulleniatina (VP.3) & - & 18,40 & - & $462,50^{*}$ & \\
\hline (f) & DRL plexo Pulleniatina (VP.2) & - & 18,80 & - & $471,93 *$ & \\
\hline (g) & DDL plexo Pulleniatina (VP.1) & 16,20 & 19,15 & $481,79^{*}$ & 480,18 & \\
\hline
\end{tabular}

\section{RESULTADOS}

A análise bioestratigráfica realizada nos testemunhos BS-C e BS-D (Figuras 3,4) permitiu o reconhecimento de sete zonas de Ericson \& Wollin (1968), estas representando intervalos glaciais (zonas $\mathrm{U}, \mathrm{W}$ e $\mathrm{Y}$ ) e interglaciais (zonas $\mathrm{T}, \mathrm{V}$ e X) do Pleistoceno e o Holoceno (Zona Z), cobrindo os últimos $\sim 620$ ka (Tabela
4). O refinamento destas zonas permitiu o reconhecimento de 14 subzonas (Tabela 4), a divisão da subzona V2 em V2B e V2A e a identificação de oito horizontes de correlação baseados no plexo Pulleniatina. As zonas/subzonas foram reconhecidas em ambos os testemunhos, com exceção da zona $\mathrm{T}$ presente apenas no testemunho BS-D (Figuras 3, 4), permitindo assim a correlação entre as seções (Figura 5). 
Tabela 4. Ciclos climáticos, zonas de Ericson \& Wollin (1968) e subzonas identificadas nos testemunhos BS-C e BS-D, profundidade e taxa de acumulação estimada para as zonas e subzonas e referências utilizadas. Abreviaturas: (a) Ericson \& Wollin (1968); Vicalvi (1997, 1999); Sanjinés (2006); Portilho-Ramos et al. (2006); (b) Vicalvi (1997, 1999); Sanjinés (2006); (c) Vicalvi (1997, 1999); Sanjinés (2006); PortilhoRamos et al. (2006); (d) Vicalvi (1997, 1999); Sanjinés (2006); (e) Kennett \& Huddlestun (1972); Vicalvi (1997, 1999); Sanjinés (2006); (f) Neff (1985); Martin et al. (1990, 1993); (g) Novas subzonas (este trabalho); (h) Ericson \& Wollin (1968); Neff (1985); Martin et al. (1990, 1993).

Table 4. Climatic cycles, Ericson \& Wollin (1968) zonation and subzones indentified on cores BS-C and BS-D, depth and estimated accumulation rate for the zones and subzones and references used.

\begin{tabular}{|c|c|c|c|c|c|c|c|c|}
\hline & \multicolumn{4}{|c|}{ Ciclos climáticos e zonas de Ericson \& Wollin (1968) e subzonas } & \multicolumn{2}{|c|}{ Testemunho (m) } & \multicolumn{2}{|c|}{ Taxa de acumulação $(\mathrm{cm} / \mathrm{ka})$} \\
\hline & \multirow{12}{*}{ 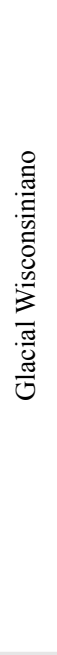 } & & & & BS-C & BS-D & BS-C & BS-D \\
\hline (a) & & Pós-glacial & & Z & $0-0,25$ & $0-0,22$ & 2,27 & 2,00 \\
\hline (b) & & \multirow{3}{*}{ Último Máximo Glacial } & \multirow{3}{*}{$\mathrm{Y}$} & Y1A & $0,25-0,55$ & $0,22-0,58$ & 7,50 & 9,00 \\
\hline (b) & & & & Y1B & $0,55-0,90$ & $0,58-1,25$ & 1,17 & 2,23 \\
\hline (c) & & & & Y2 & $0,90-2,37$ & $1,25-4,25$ & 3,27 & 6,67 \\
\hline (d) & & \multirow[t]{4}{*}{ Interestadial } & \multirow[t]{2}{*}{$X$} & $\mathrm{X} 2$ & $2,78-3,80$ & $4,85-5,46$ & \multirow[t]{2}{*}{4,20} & \multirow[t]{2}{*}{3,80} \\
\hline (d) & & & & $\mathrm{X} 3$ & $3,80-4,30$ & $5,46-6,00$ & & \\
\hline (e) & & & \multirow{2}{*}{ W } & W1 & $4,30-4,74$ & $6,00-6,45$ & \multirow{2}{*}{1,36} & \multirow{2}{*}{2,43} \\
\hline (e) & & & & W2 & $4,74-5,06$ & $6,45-7,36$ & & \\
\hline (f) & & \multirow{3}{*}{ Interglacial Sangamom } & \multirow{3}{*}{$\mathrm{V}$} & V1 & $5,06-8,25$ & $7,36-11,65$ & \multirow{2}{*}{3,76} & \multirow{2}{*}{3,92} \\
\hline (g) & & & & $\mathrm{V} 2 \mathrm{~B}$ & $11,50-13,10$ & $14,93-15,75$ & & \\
\hline (f) & & & & $\mathrm{V} 3$ & $13,10-16,32$ & $15,75-79,35$ & 3,79 & 4,24 \\
\hline (f) & & \multirow{2}{*}{ Glacial Illinoian } & \multirow{2}{*}{$\mathrm{U}$} & U1 & $16,32-16,88$ & $19,35-19,94$ & \multirow{2}{*}{2,82} & \multirow{2}{*}{1,49} \\
\hline (f) & & & & $\mathrm{U} 2$ & $16,88-19,84$ & $19,94-21,21$ & & \\
\hline (h) & & Interglacial Yarmouth & & $\mathrm{T}$ & $19,84-20,43$ & - & - & - \\
\hline
\end{tabular}

\section{Zonas e Subzonas}

Zona T - Pleistoceno (Tabelas 2, 4; Figura 6): reconhecida apenas no testemunho BS-C, onde compreende o intervalo entre a base $(20,43 \mathrm{~m})$ e a profundidade $19,84 \mathrm{~m}$. A Zona T é caracterizada pela presença do plexo $G$. menardii, onde se destaca $G$. menardii seguida de G. tumida, enquanto G. menardii flexuosa é ausente. O plexo Pulleniatina está presente, enquanto $N$. dutertrei e $G$. truncatulinoides estão representadas com algum significado na base do testemunho. As demais espécies, G. inflata, G. crassaformis, $G$. conglobatus e $O$. universa, são raras ou ausentes. O limite T/U é marcado pelo desaparecimento do plexo G. menardii, seguido pelo aumento do plexo Pulleniatina e de G. inflata. Zona U - Pleistoceno (Tabelas 2, 4; Figura 6): presente no BS-C, enquanto no testemunho BS-D compreende o intervalo entre a base $(21,21 \mathrm{~m})$ e a profundidade de $19,35 \mathrm{~m}$ (Figura 4). A zona U é caracterizada pela ausência do plexo G. menardii e constante presença do plexo Pulleniatina, este último registrado ao longo de todo o intervalo. G. inflata e $N$. dutertrei apresentam picos de abundância próximos aos limites inferior (T/U) e superior (U/V). G. truncatulinoides está representada ao longo de todo o intervalo, com as maiores abundâncias ocorrendo no centro da zona. G. crassaformis é constante e ocorre com maior representatividade próxima ao limite U2/U1, enquanto G. conglobatus apresenta seu pico de abundância após o limite T/U. O limite U2/U1 foi estimado entre 501,64 e 499,78 ka (BS-C e BS-D) e está marcado pela diminuição do plexo Pulleniatina e da espécie G. inflata, ambas mais frequentes na Subzona U2. Na Subzona U1 o plexo Pulleniatina passa a ser raro, seguido pela diminuição de G. inflata, G. conglobatus e G. truncatulinoides. A alteração no sentido de enrolamento da espécie $G$. truncatulinoides foi utilizada para a definição do limite entre as subzonas (U2/U1), onde a Subzona U2 é marcada pelo predomínio de espécimes sinistrais, enquanto na U1 predominam espécimes dextrais. Zona V - Pleistoceno (Tabelas 2, 4; Figura 6): zona mais espessa identificada (média 11,62 m). A Zona V é caracterizada por grandes oscilações dos plexos G. menardii e Pulleniatina, este último marcando a região basal da zona com frequentes desaparecimentos e reaparecimentos. As espécies G. truncatulinoides e G. inflata foram utilizadas de forma complementar às observações dos plexos G. menardii e Pulleniatina, para a divisão da Zona V nas subzonas V3, V2 e V1 e para a subdivisão da Subzona V2 em V2B e V2A (Figuras 3, 4, 6).

A Subzona V3 é marcada pelo reaparecimento do plexo $G$. menardii (limite $\mathrm{V} 3 / \mathrm{U}$ ) onde $G$. menardii é a principal espécie seguida de $G$. tumida, enquanto $G$. menardi flexuosa é rara ou ausente. G. truncatulinoides e G. inflata apresentam seus maiores picos de abundância próximos ao limite inferior (U1/V3) e superior (V3/V2). Os frequentes desaparecimentos e reaparecimentos do plexo Pulleniatina são a principal característica da Subzona V3. As oscilações do plexo permitiram a definição de um conjunto de horizontes utilizados para a correlação entre os testemunhos (Tabela 3). Os biohorizontes identificados foram denominados de horizontes de correlação VP., onde os ímpares (VP.1, VP.3, $V P .5$ e $V P .7)$ representam eventos de desaparecimento local e os pares (VP.2, VP.4, VP.6 e VP.8) de reaparecimento local do plexo Pulleniatina. 


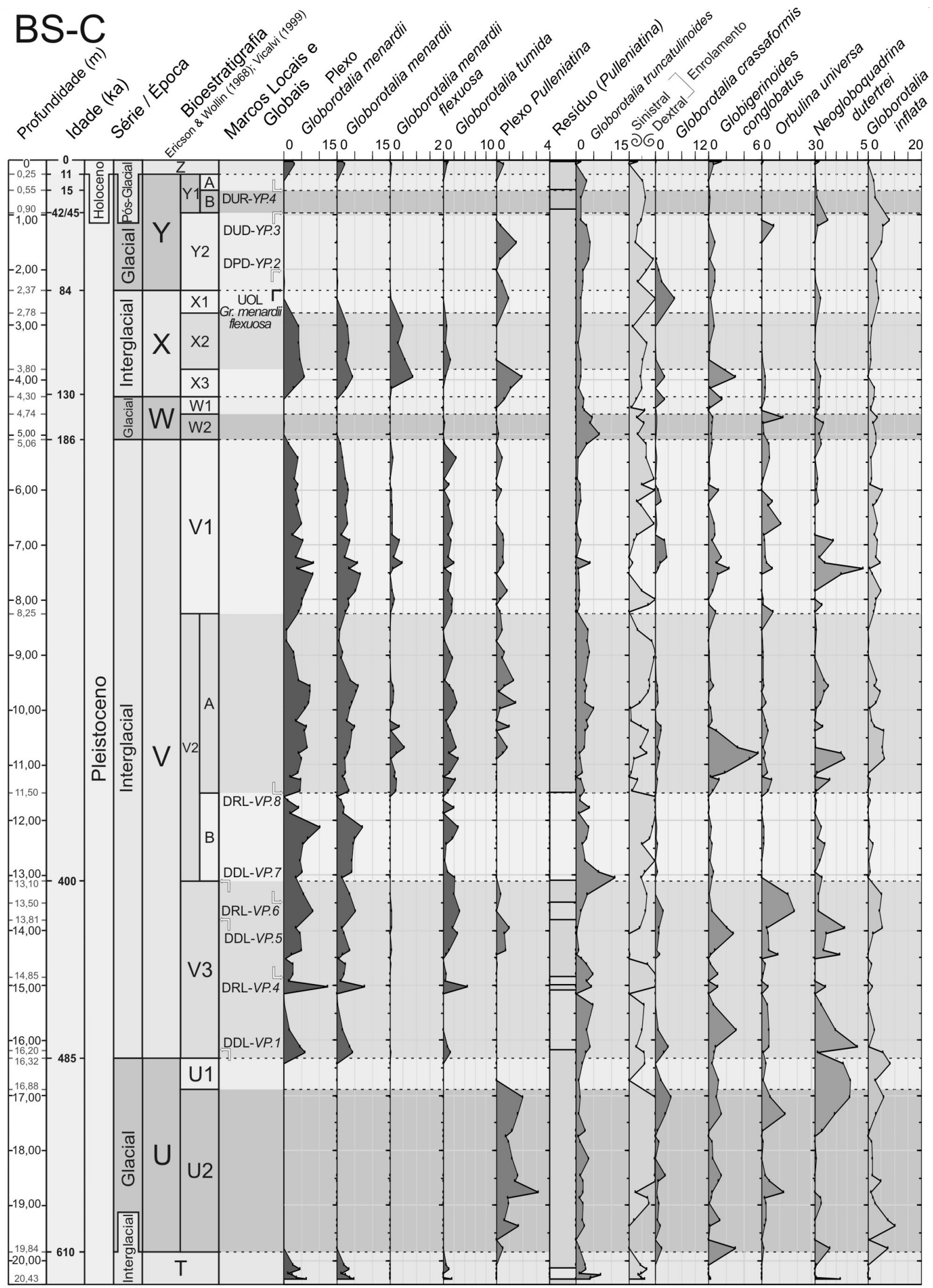

Figura 3. Testemunho BS-C: bioestratigrafia baseada no zoneamento climático de Ericson \& Wollin (1968); datums globais (Damuth, 1975) e locais (Vicalvi, 1997, 1999; novos horizontes de correlação); e abundância relativa das espécies de foraminíferos planctônicos selecionadas.

Figure 3. Core BS-C: biostratigraphy based on climatic zonation of Ericson \& Wollin (1968); global (Damuth, 1975) and local datums (Vicalvi, 1997, 1999; new data); relative abundance of selected planktonic foraminifera species. 
O primeiro desaparecimento observado ocorre logo após a chegada do plexo G. menardii (limite U1/V3) na base da Subzona V3. Utilizado como Datum de Desaparecimento Local (DDL) e denominado de biohorizonte VP.1, teve sua posição ajustada ao topo da fácies MG (marga; BS-C: 16,20 m; BS-D: 19,15 m) e idade estimada entre 481,79 e 480,18 ka (testemunhos BS-C e BS-D respectivamente; Tabela 3) com base na taxa de acumulação da subzona (Tabela 4). O primeiro reaparecimento e segundo desaparecimento do plexo foram observados apenas no testemunho BS-D. Associado ao contato entre as fácies LL (lama levemente carbonática) e LR (lama rica em carbonatos; 18,80 m) e com idade estimada em $471,93 \mathrm{ka}$ (Tabela 3), o reaparecimento do plexo foi definido como biohorizonte VP.2 (Datum de Reaparecimento Local - DRL). O desaparecimento ocorre no centro da fácies LR $(18,40 \mathrm{~m})$, definido como biohorizonte VP.3 (DDL) e teve sua idade estimada em 462,50 ka (Tabela 3). Neste intervalo pode se observar no testemunho BS-C sinais de pelo menos dois eventos de remobilização sedimentar, estes marcados por intercalação de pacotes arenosos e lamas ricas em carbonatos (Figura 5) com elevadas abundância de formas bentônicas, sugerindo que a ausência dos horizontes de correlação VP.2 e VP.3 possa estar relacionada a estes eventos.

Associado ao contato entre a fácies LR e um pacote arenoso (AS - areia siliciclástica), ocorre um novo reaparecimento do plexo Pulleniatina na Subzona V3 (biohorizonte VP.4; BS-C: 14,85 m; BS-D: 17,91 m) com idade estimada entre 446,17 e 450,94 ka (BS-C e BS-D) o qual foi utilizado como horizonte de correlação (DRL). Após o seu reaparecimento, os plexos (G. menardii e Pulleniatina) podem apresentar um ou mais picos de abundância, onde os maiores valores do plexo Pulleniatina são observados (BS-C: 0,90\% - 14,07 m; BS-D: $3,54 \%-17,50 \mathrm{~m}$ ). A espécie G. truncatulinoides apresenta domínio de espécimes dextrais e os menores valores nessa subzona. $G$. inflata apresenta um comportamento similar, apresentando neste intervalo os menores valores observados para a Subzona V3. O terceiro desaparecimento do plexo está associado à fácies LL (BS-C: 13,81 m; BS-D: 16,95 m), denominado biohorizonte VP.5 (DDL) com idade estimada entre 416,74 e 428,30 ka (BS-C e BS-D).

O terceiro reaparecimento do plexo (VP.6; Tabela 3) ocorre próximo ao topo da Subzona, com idade estimada entre 410,55 e 407,07 ka (BS-C e BS-D). Este evento está associado à fácies LL no testemunho BS-C $(13,50 \mathrm{~m})$ e à fácies LR no testemunho BS-D (16,05 m). O intervalo entre os biohorizontes VP.5 e VP.6 é marcado pelo aumento gradual na abundância de $G$. truncatulinoides e $G$. inflata, enquanto o plexo G. menardii diminui gradativamente sua expressão. O último desaparecimento do plexo (VP.7; Tabela 3) ocorre junto ao limite V3/V2, com idade estimada entre 399,83 e 400,03 ka (BS-C e BS-D) e está associado ao contato entre as fácies LL e MG no testemunho BS-C (13,10 m) e ao centro da fácies LR no testemunho BS-D $(15,75 \mathrm{~m})$. Associado ao $V P .7$ ocorrem as menores abundâncias do plexo G. menardii junto a picos de abundância de G. truncatulinoides, sendo este critério utilizado para a definição do limite V3/V2.

A Subzona V2 é marcada pela constante presença do plexo
G. menardii, onde G. menardii é a principal componente, seguida de G. tumida, enquanto $G$. menardii flexuosa é rara ou ausente. A base da Subzona é marcada pela ausência de $G$. menardii flexuosa e do plexo Pulleniatina, que reaparecem próximo ao meio da sequência. O reaparecimento do plexo Pulleniatina (biohorizonte VP.8) teve sua idade estimada entre 357,27 e 379,11 ka (BS-C e BS-D). A ausência do plexo Pulleniatina e da espécie G. menardii flexuosa na base da Subzona V2 foram utilizadas como critério para a divisão da Subzona V2 em V2B e V2A. Assim, a Subzona V2B é caracterizada principalmente pela ausência do plexo Pulleniatina e da espécie G. menardii flexuosa. De forma complementar, foi observado o domínio de espécimes com enrolamento sinistral de $G$. truncatulinoides e a diminuição em sua abundância após o limite V3/V2, seguida por um ligeiro aumento próximo ao limite $\mathrm{V} 2 \mathrm{~B} / \mathrm{V} 2 \mathrm{~A}(V P .8)$. O plexo $G$. menardii aumenta sua abundância no sentido base-topo desta Subzona, sofrendo uma queda brusca no topo da mesma. Esta queda ocorre junto ao retorno do plexo Pulleniatina, marcando o limite biohorizonte VP.8 (limite V2B/V2A). Após o retorno (VP.8; V2B/V2A), o plexo pode apresentar picos de abundância até o limite superior da Zona V (V/W). $G$. menardii flexuosa apresenta comportamento similar, ao longo da Subzona V2A, no entanto torna a desaparecer próximo ao limite entre as subzonas V2A/V1. O plexo G. menardii volta a apresentar aumento em sua abundância, atingindo valores mais elevados no centro da subzona e reduzindo sua expressão até o limite V2A/V1, onde é pouco representativo. G. inflata pode apresentar um ou mais picos de abundância na metade da Subzona V2A, diminuindo sua expressão próximo ao limite V2A/V1. A idade para o limite V2A/V1 (Tabela 2) foi estimada entre 270,84 e 295,44 ka (BS-C e BS-D) e seu posicionamento definido com base nos menores valores do plexo G. menardii, seguido de uma redução nos valores de G. truncatulinoides e uma ligeira diminuição de G. inflata.

A Subzona V1 é caracterizada pela constante presença dos plexos G. menardii e Pulleniatina. G. truncatulinoides está presente ao longo de todo intervalo, apresentando valores menores quando comparada com as demais (V3, V2B e V2A). Próximo ao topo da Zona V (Subzona V1), a espécie apresenta um pico de abundância que auxilia o reconhecimento do limite V1/W2. G. inflata também aparece de forma constante, podendo apresentar um ou mais picos de abundância ao longo do intervalo. O limite V/W (V1/ W2) é marcado pelo desaparecimento do plexo G. menardii, enquanto $G$. truncatulinoides e $G$. inflata aumentam sua abundância na base da Zona W.

Zona W - Pleistoceno (Tabelas 2, 4; Figura 6): caracterizada pela ausência ou baixa representatividade do plexo $G$. menardii, enquanto o plexo Pulleniatina é raro ou ausente na região basal. G. truncatulinoides pode apresentar um pico de abundância próximo ao centro do intervalo, diminuindo em direção ao topo onde ocorrem as menores abundâncias. Este pico ocorre próximo ao retorno do plexo Pulleniatina, no testemunho BS-D e foi utilizado de forma complementar para identificar o limite entre as subzonas W2/W1. G. inflata, G. conglobatus e $O$. universa estão presentes ao longo de toda 


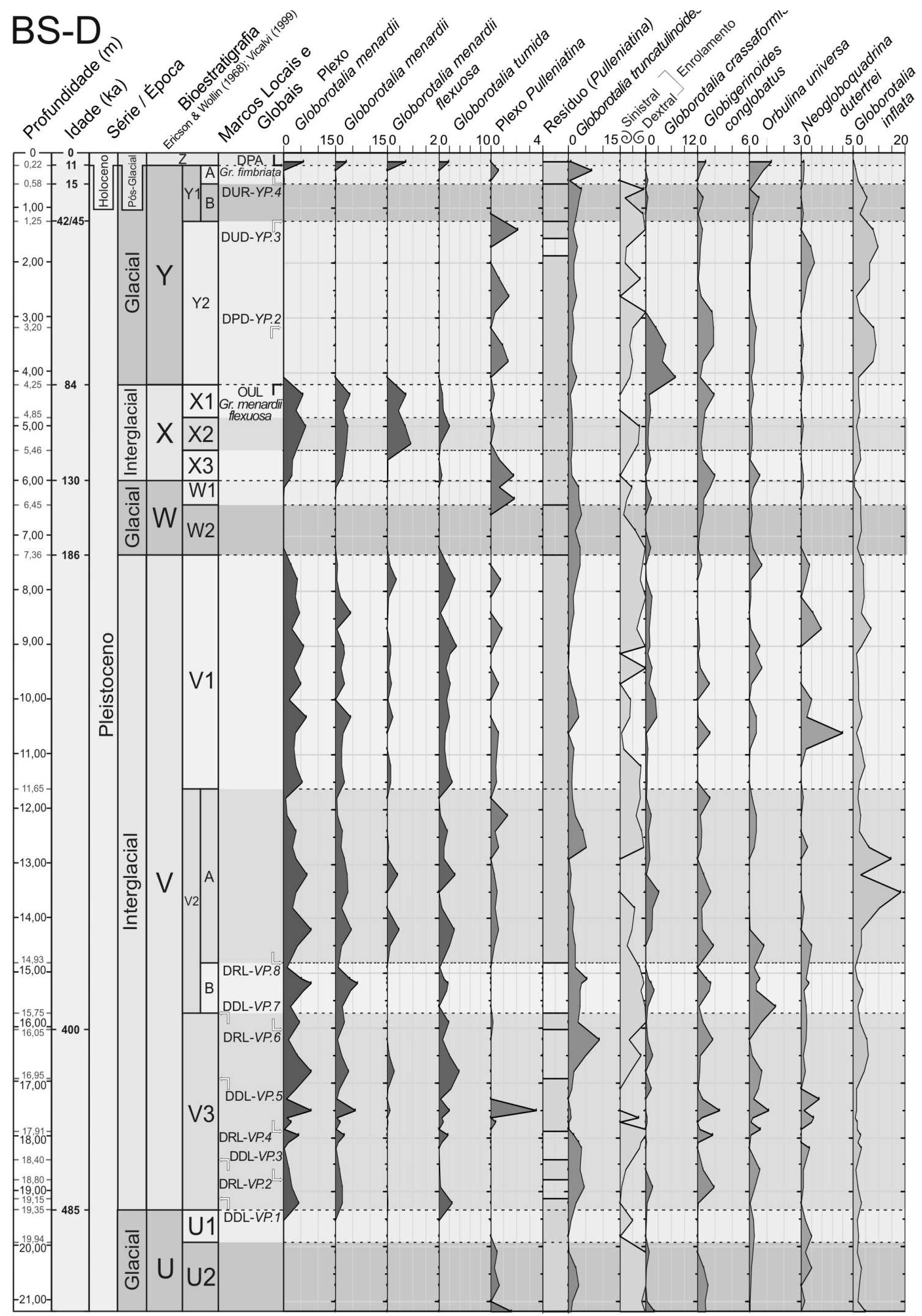

Figura 4. Testemunho BS-D: bioestratigrafia baseada no zoneamento climático de Ericson \& Wollin (1968); datums globais (Bolli \& Premoli Silva, 1973; Damuth, 1975) e locais (Vicalvi, 1997, 1999; novos horizontes de correlação); e abundância relativa das espécies de foraminíferos planctônicos selecionadas.

Figure 4. Core BS-D: biostratigraphy based on climatic zonation of Ericson \& Wollin (1968); global (Bolli \& Premoli Silva, 1973; Damuth, 1975) and local datums (Vicalvi, 1997, 1999; new data); and relative abundance of selected planktonic foraminifera species. 
a zona, podendo apresentar um ou mais picos de abundância ao longo do intervalo. G. crassaformis e $N$. dutertrei são raras, mas podem apresentar picos de abundância. O limite W2/W1 é marcado por um abrupto aumento de G. inflata seguido de decréscimo de $N$. dutertrei. A identificação deste comportamento, complementado pelas oscilações apresentadas por G. truncatulinoides e pelo plexo Pulleniatina (ausente no testemunho BS-D e raro no testemunho BS-C) auxiliaram o posicionamento do mesmo. A idade estimada para o limite oscilou entre 162,35 e 148,52 ka (Tabela 2).

A Subzona W2 é marcada pela ausência (BS-D) ou baixa representatividade (BS-C) dos plexos G. menardii e Pulleniatina (presente apenas no testemunho BS-C), enquanto G. truncatulinoides apresenta seu pico de abundância próximo ao topo da subzona, diminuindo após o limite W2/W1. A Subzona W1 é marcada pelo aumento do plexo Pulleniatina, concomitante com uma pequena diminuição de G. truncatulinoides. G. crassaformis e G. conglobatus apresentam as maiores abundâncias neste intervalo, enquanto $N$. dutertrei e $O$. universa são raras ou ausentes. O limite entre as zonas $\mathrm{W} / \mathrm{X}(\mathrm{W} 1 / \mathrm{X} 3)$ é marcado pelo retorno do plexo G. menardii, seguido do aumento do plexo Pulleniatina e decréscimo de $G$. inflata e $G$. truncatulinoides.

Zona X - Pleistoceno (Tabelas 2, 4; Figura 6): caracterizada pela constante presença do plexo G. menardii, em especial a espécie G. menardii flexuosa (Tabela 2). O plexo Pulleniatina e $O$. universa apresentam maior significado na base da zona, enquanto $G$. conglobatus pode apresentar seu pico de abundância na base (BS-C e BS-D) e/ou no topo (BS-D) do intervalo. As alterações no sentido do enrolamento de $G$. truncatulinoides, que está representada ao longo de toda a Zona, foram utilizadas de forma complementar para a definição dos limites entre as subzonas (X3, X2 e X1). G. crassaformis e G. inflata apresentam comportamento similar, onde as menores abundâncias são observadas na base da zona, seguido por um pequeno aumento. Utilizando a variação observada nos plexos G. menardii (espécie G. menardii flexuosa) e Pulleniatina e nas espécies G. inflata, G. crassaformis e $G$. truncatulinoides, complementada pela observação no sentido de enrolamento desta última, a Zona $\mathrm{X}$ pode ser dividida em três subzonas (X3, X2 e X1). A Subzona X3 é marcada pela baixa representatividade de G. menardii flexuosa, enquanto o plexo Pulleniatina e as espécies $G$. conglobatus e $O$. universa apresentam pico de abundância. G. truncatulinoides está presente ao longo de todo intervalo com domínio de espécimes dextrais. Exceção a esta observação ocorre no testemunho BS-C, onde os espécimes apresentam aparente equilíbrio entre os sentidos de enrolamento. N. dutertrei e G. crassaformis podem apresentar um pico de abundância próximo à base da subzona, enquanto $G$. inflata aumenta sua abundância logo após o limite inferior. O limite X3/X2 é marcado pelo aumento na abundância de G. menardii flexuosa, seguido pela diminuição na abundância do plexo Pulleniatina, de G. conglobatus e $O$. universa. De forma complementar observou-se a alteração no sentido de enrolamento de G. truncatulinoides passando de dextral para sinistral. Este limite teve sua idade estimada entre 118,08 e 115,79 ka (BS-C e BS-D).
Ao longo da Subzona X2, G. menardii flexuosa aparece de forma mais expressiva, enquanto $G$. truncatulinoides diminui sua abundância e apresenta domínio de indivíduos sinistrais. $\mathrm{O}$ plexo Pulleniatina e as espécies $O$. universa e G. conglobatus aparecem com as menores abundâncias. G. crassaformis é constante, enquanto $N$. dutertrei apresenta um pequeno pico de abundância no topo da subzona, concomitante a uma pequena queda na abundância de $G$. inflata. O limite X2/X1 teve idade estimada entre 93,81 e 99,74 (BS-C e BS-D) e está marcado pela diminuição da abundância de G. menardii flexuosa, seguida por alteração no sentido de enrolamento de $G$. truncatulinoides e aumento na abundância de G. conglobatus. A Subzona X1 é marcada pela menor expressão do plexo $G$. menardii quando comparada à X2. $\mathrm{O}$ desaparecimento do plexo, em especial da G. menardii flexuosa no topo da subzona marca o limite X/Y (X1/Y). O plexo Pulleniatina volta a apresentar abundâncias mais elevadas quando comparada à Subzona X2 no testemunho BS-C, enquanto no testemunho BS-D é raro. G. truncatulinoides apresenta comportamento estável ao longo da zona, voltando a apresentar domínio de espécimes com enrolamento dextral na base da subzona no testemunho BS-D, enquanto no BS-C ocorre o domínio de indivíduos com enrolamento sinistral. G. inflata apresenta ligeiro aumento em sua abundância, enquanto G. conglobatus pode apresentar um pico de abundância (BS-D; Figura 4) próximo ao limite superior $(\mathrm{X} / \mathrm{Y})$. O. universa, $N$. dutertrei e G. crassaformis são raras ou ausentes. Exceção ocorre no testemunho BS-C onde a última espécie apresenta um pico de abundância próximo ao limite superior.

$\mathrm{O}$ limite X1/Y é marcado pelo desaparecimento do plexo G. menardii, principalmente a espécie G. menardii flexuosa (Tabela 2). O plexo Pulleniatina e G. conglobatus diminuem sua abundância após o limite, enquanto G. truncatulinoides, $G$. crassaformis e $G$. inflata aumentam.

Zona Y - Pleistoceno (Tabelas 2, 4; Figura 6): zona marcada pela ausência ou baixa representatividade do plexo $G$. menardii e pelas oscilações do plexo Pulleniatina. As espécies $G$. truncatulinoides e $G$. inflata estão presentes em toda a zona com valores significativos, enquanto $G$. crassaformis e G. conglobatus apresentam elevadas abundâncias na região basal. $N$. dutertrei é mais bem representada próximo à base da zona (biohorizonte YP.3). A partir das oscilações do plexo Pulleniatina pode-se reconhecer três horizontes de correlação (Tabela 3), sendo eles YP.2, YP.3 (limite Y2/Y1B) e YP.4 (limite Y1B/Y1A). O reconhecimento dos biohorizontes fornecidos pelo plexo Pulleniatina permitiu a divisão da Zona Y em três subzonas (Y2, Y1B e Y1A).

A Subzona Y2 teve seu limite inferior (X/Y2) definido com base no desaparecimento do plexo $G$. menardii (UOL $G$. menardii flexuosa; Tabela 3) e seu limite superior (Y2/Y1B) posicionado com base no biohorizonte YP.3 (Figuras 3, 4). Esta Subzona (Y2) é caracterizada pela presença constante do plexo Pulleniatina, que pode apresentar um ou mais picos de abundância ao longo deste intervalo. O biohorizonte YP.2 foi identificado no contato entre as fácies LL e LR no testemunho BS-C (2,02 m) e na fácies LL no testemunho BS-D (3,20 m). $G$. inflata e $G$. truncatulinoides mostram comportamento 


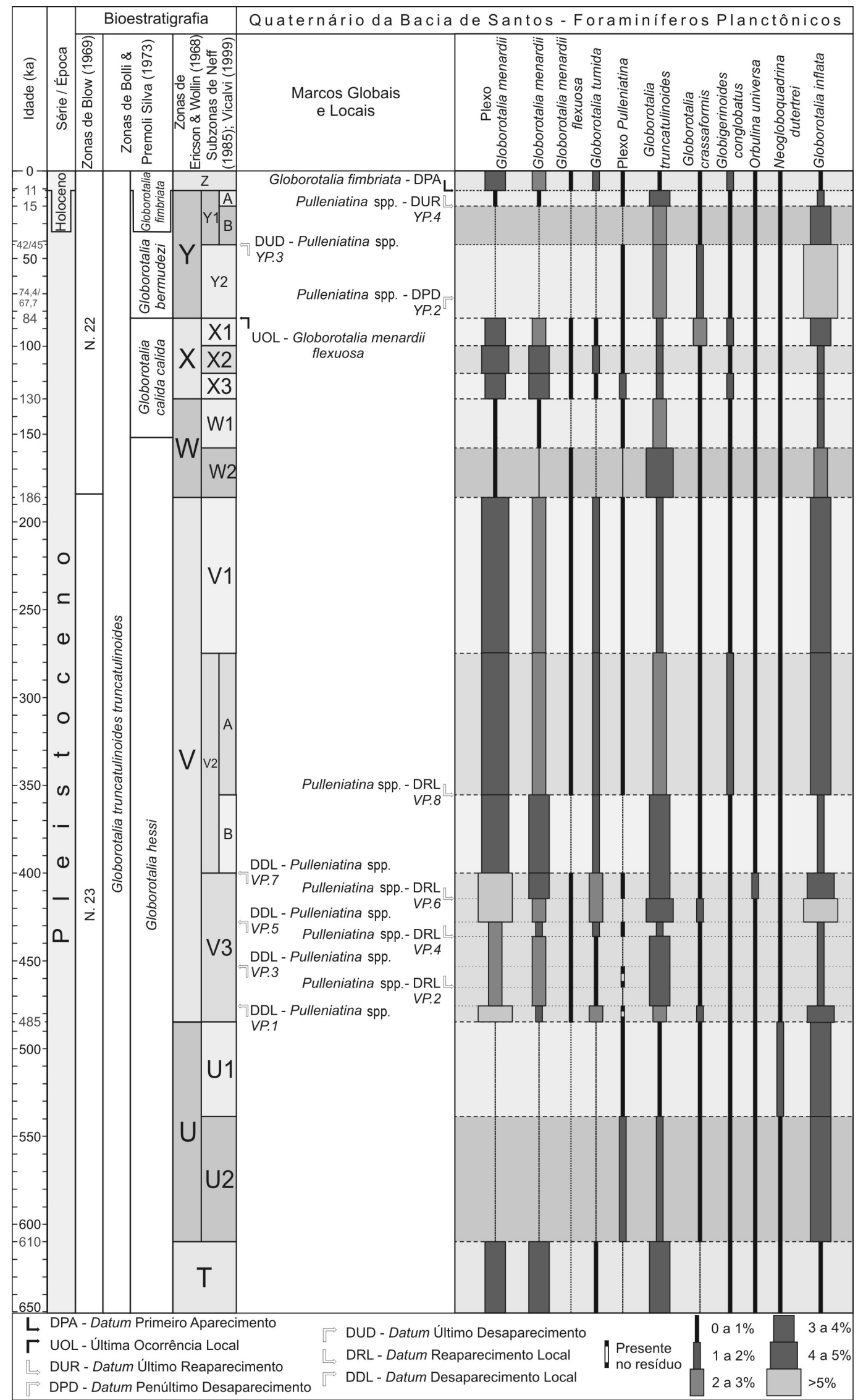

Figura 5. Arcabouço biocronoestratigráfico do Quaternário da bacia de Santos e abundância relativa média (\%) das principais espécies.

Figure 5. Quaternary chronobiostratigraphy chart of Santos Basin and mean relative abundances (\%) of main species. 


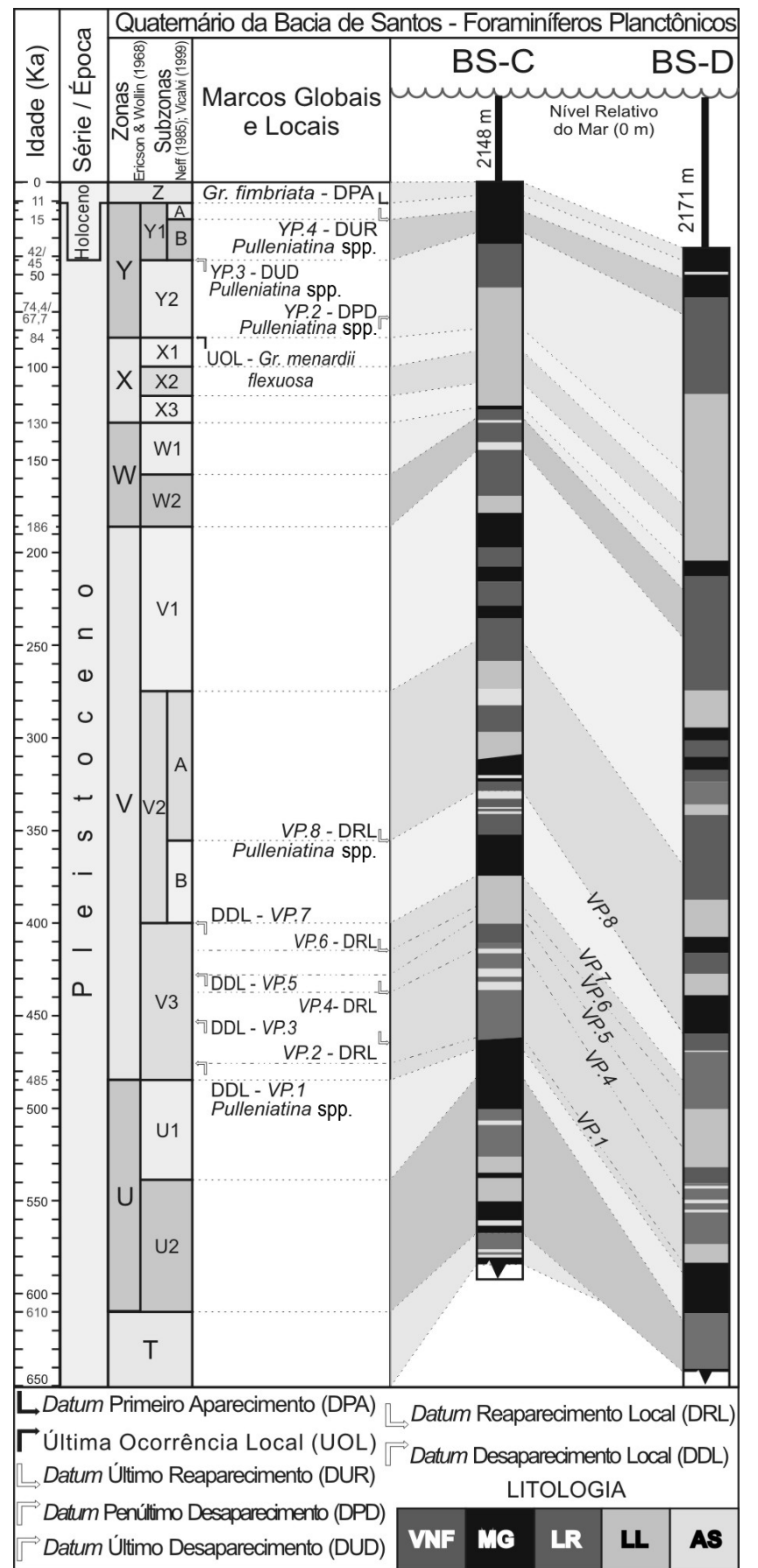

Figura 6. Correlação entre os testemunhos BS-C e BS-D.

Figure 6. Correlation between piston cores BS-C and BS-D.

similar ao longo da Subzona Y2, podendo apresentar um ou mais picos de abundância, que ocorrem na base deste intervalo no testemunho BS-D, enquanto no topo (Y1B/Y1A) ocorre em ambos os testemunhos (BS-C e BS-D). G. crassaformis apresenta maior significado na parte basal do intervalo, enquanto $G$. conglobatus aparece de forma mais significativa na parte basal do testemunho BS-D, e no meio da sequência, no testemunho BS-C. $N$. dutertrei e $O$. universa apresentam algum significado próximo ao topo da Subzona. O limite Y2/ Y1B é marcado pelo desaparecimento do plexo Pulleniatina (biohorizonte YP.3; Tabela 3).
A Subzona Y1B é caracterizada pela ausência do plexo Pulleniatina. Seu limite inferior (Y2/Y1B) é marcado pelo biohorizonte YP.3, enquanto o superior (Y1B/Y1A) é marcado pelo biohorizonte YP.4 (Tabela 3). G. inflata e $G$. truncatulinoides apresentam queda constante na sua abundância ao longo do intervalo, com menores valores quando comparados à Subzona Y2. No testemunho BS-C, o comportamento de G. truncatulinoides é oposto ao observado no testemunho BS-D, aumentando sua abundância ao longo deste intervalo. As espécies $G$. crassaformis, $N$. dutertrei e $O$. universa são raras ou ausentes, enquanto $G$. conglobatus diminui sua abundância em relação à Subzona Y2. A Subzona Y1A é caracterizada pelo regresso do plexo Pulleniatina. Seu limite inferior é marcado pelo biohorizonte YP.4 e o limite superior (Y1A/Z) é marcado pelo regresso do plexo G. menardii que marca a passagem Pleistoceno/Holoceno (Tabelas 3, 4). Nesta Subzona, G. inflata e G. truncatulinoides demonstram um comportamento inverso, onde $G$. inflata diminui e $G$. truncatulinoides aumenta sua abundância. As demais espécies, G. crassaformis e G. conglobatus são raras ou ausentes, enquanto O. universa e $N$. dutertrei podem apresentar algum significado.

Zona Z-Holoceno (Tabelas 2, 4; Figura 6): marca o regresso do plexo G. menardii, onde se destaca $G$. menardii seguida de G. tumida. A presença de G. fimbriata no testemunho BS-D confirma a idade holocênica atribuída aos sedimentos (Tabela 3). Concomitante ao regresso do plexo G. menardii há uma diminuição na abundância de $G$. inflata e $G$. truncatulinoides. As demais espécies analisadas apresentam baixos percentuais, sendo as mais abundantes $O$. universa

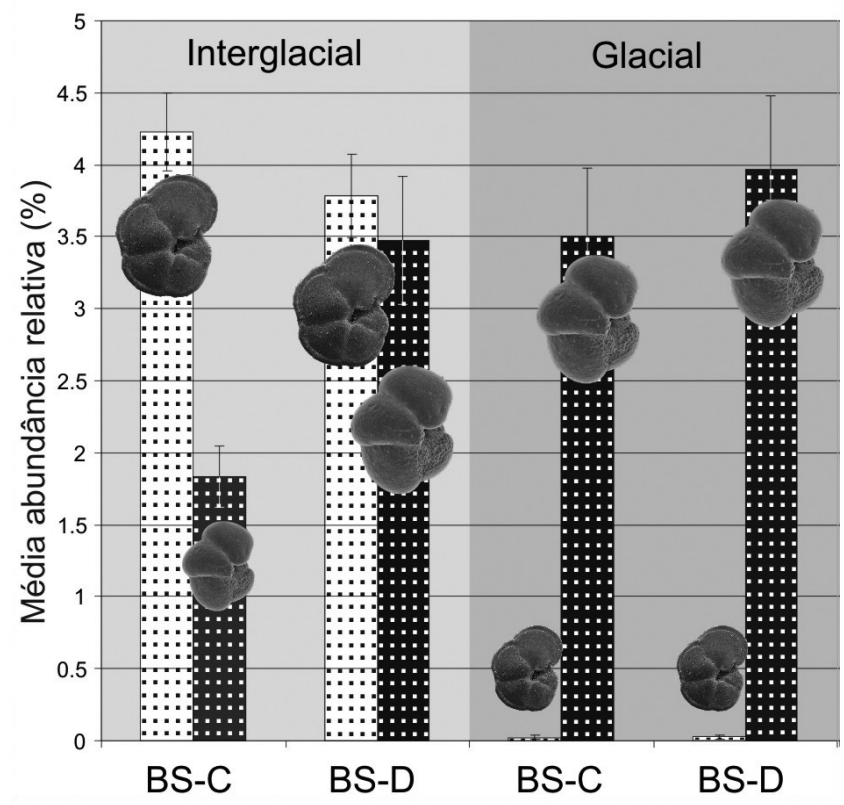

Figura 7. Abundância relativa média (\%) do plexo Globorotalia menardii e da espécie Globorotalia inflata ao longo dos intervalos interglaciais e glaciais reconhecidos nos testemunhos BS-C e BS-D da bacia de Santos.

Figure 7. Mean relative abundance (\%) of plexo Globorotalia menardii and Globorotalia inflata along the interglacial and glacial intervals recognized in cores BS-C and BS-D of the Santos Basin. 
seguida de G. conglobatus, enquanto G. crassaformis e $N$. dutertrei são raras ou ausentes.

\section{DISCUSSÃO}

O zoneamento climático estabelecido demonstrou que as seções estudadas registram as oscilações climáticas dos últimos $\sim 620 \mathrm{ka}$ (BS-C: 620 ka; BS-D: 530 ka). A partir da variação na abundância relativa do conjunto de espécies selecionadas, em especial dos plexos Globorotalia menardii e Pulleniatina, foi possível reconhecer zonas e subzonas (Figura 6) pouco conhecidas e/ou ainda não descritas para a margem continental brasileira (zonas T, U e V; subzonas V3, V2, V1, U2 e U1) e refinar a Subzona V2, com sua divisão nas subzonas V2B e V2A. Estas zonas/subzonas, excetuando a Zona T, presente apenas no testemunho BS-C (Figuras 3, 4), foram reconhecidas em ambos os testemunhos, permitindo uma correlação mais precisa entre as seções (Figura 5) e sugerindo a continuidade das mesmas. O comportamento marcado por constantes desaparecimentos e reaparecimentos do plexo Pulleniatina ao longo das subzonas V3 e V2 permitiu a definição de oito biohorizontes, refinando o zoneamento de cada testemunho e sua correlação.

O intervalo mais antigo recuperado está representado pela Zona T de Ericson \& Wollin (1968). Com duração aproximada de $380 \mathrm{ka}$ (Thunell, 1984), somente uma pequena parte deste interglacial foi recuperada na base do testemunho BS-C ( 0,60 m; Figura 3). Representa um intervalo climático quente, evidenciado pela presença do plexo G. menardii e a baixa representatividade de $G$. inflata (Figura 6). O desaparecimento do plexo G. menardii seguido pelo aumento de G. inflata marcam a passagem da Zona T (interglacial) para a Zona U (glacial). Hall et al. (2001) atribuem a este período uma diminuição na produção da APAN e o aumento na produção de massas d'águas no Sul, sugerindo que o limite T/U marca a chegada de águas frias vindas desta região, o que pode provocar alterações na estrutura da lâmina d'água. A Zona U tem duração aproximada de 125 ka e está marcada pela constante presença do plexo Pulleniatina, em especial ao longo da Subzona U2 onde são observadas as maiores abundâncias. A presença do plexo Pulleniatina junto a $G$. inflata e $G$. truncatulinoides (sinistral) sugere que a região foi controlada por águas transicionais ao longo deste intervalo. O domínio de espécimes dextrais de G. truncatulinoides e a maior abundância de $N$. dutertrei sugerem que ao longo da Subzona U1 houve um aumento na produtividade local, em especial do fitoplâncton, uma vez que as diatomáceas são a principal fonte de alimento de $N$. dutertrei (Hemleben et al., 1989; Hilbrecht, 1997; Toledo et al., 2007). Esta maior produção de fitoplâncton pode ter sido a responsável pela queda na abundância do plexo Pulleniatina observada no limite U2/U1. A alteração no sentido de enrolamento de $G$. truncatulinoides observada no limite U2/U1 também foi utilizada como critério bioestratigráfico em seções do Golfo do México (Neff, 1985; Martin et al., 1990, 1993).

O retorno do plexo G. menardii marca a chegada do interglacial representado pela Zona V. Com duração aproximada de $299 \mathrm{ka}$, a Zona V é marcada por grandes oscilações climáticas e engloba os estágios isotópicos marinhos (Marine Isotopic Stage - MIS) de 7 a 12 (Thunell, 1984; Neff, 1985; Martin et al., 1990, 1993; Kohl et al., 2004). Estas oscilações são mais bem evidenciadas pelo comportamento do plexo G. menardii (Figuras 3, 4), onde as maiores abundâncias são observadas no centro das subzonas (V3, V2 e V1) e associadas aos MIS 11, 9 e 7 respectivamente, enquanto as menores abundâncias foram utilizadas para a definição dos limites entre as subzonas (Neff, 1985; Martin et al., 1990, 1993; Martin \& Fletcher, 1995), sugerindo que estes limites estejam associados aos MIS 8 e 10 (V/2/V1 e V3/V2, respectivamente), enquanto o MIS 12 pode estar associado ao limite U/V (Kohl et al., 2004). As oscilações observadas nas espécies $G$. inflata e $G$. truncatulinoides sugerem a influência de pulsos de águas frias e/ou produtivas, auxiliando a definição dos limites entre as subzonas.

Os constantes eventos de desaparecimento e reaparecimento do plexo Pulleniatina foram utilizados para aumentar a resolução do zoneamento de cada testemunho. A constante oscilação apresentada pelo plexo, em especial ao longo da Subzona V3, não pode ser explicado neste estudo, no entanto demonstra que sua distribuição não é influenciada apenas pela temperatura (Boltovskoy et al., 1996; Schmidt et al., 2004), uma vez que este tipo de comportamento (desaparecimentos e reaparecimentos) também é observado ao longo da Zona Y (glacial) (e.g. Kennett \& Huddleston, 1972; Bé et al., 1976; Prell \& Damuth, 1978; Vicalvi, 1999; Sanjinés, 2006; Portilho-Ramos et al., 2006). Próximo ao topo da Zona V ocorre um pequeno aumento de G. truncatulinoides, seguido pelo desaparecimento do plexo G. menardii, indicando a entrada de águas frias que marcam o pequeno glacial representado pela Zona $\mathrm{W}$ ( $\sim 56 \mathrm{ka})$. O padrão apresentado pelas espécies $G$. truncatulinoides e pelos plexos $G$. menardii e Pulleniatina ao longo Zona W (Figuras 3, 4), sugere uma rápida mudança na temperatura (limite $\mathrm{V} / \mathrm{W}$ ), evidenciada pelo abrupto desaparecimento do plexo G. menardii. A baixa representatividade do plexo Pulleniatina associada aos elevados valores apresentados por $G$. truncatulinoides marcam a Subzona W2 e sugerem temperaturas mais baixas que as observadas na Subzona seguinte (W1). Na Subzona W1 ocorre um ligeiro aumento na temperatura, evidenciado pela diminuição na expressão de G. truncatulinoides seguido pelo aumento do plexo Pulleniatina.

O retorno de expressões significativas do plexo G. menardii (média 3,53\%; Figura 6) marca a base da Zona X que, por sua vez, registra a entrada de águas quentes nas quais espécies como G. truncatulinoides e G. inflata diminuem sua expressão. Durante o ligeiro aquecimento representado pela Zona X ( 46 ka), são observadas mudanças no sentido de enrolamento de G. truncatulinoides, o que pode significar mudanças na produtividade da área e/ou alterações na estrutura da coluna d'água. As subzonas X1 e X3 são dominadas por espécimes dextrais, sugerindo que ao longo destas subzonas ocorreu uma maior produtividade no local, enquanto a Subzona X2 é dominada por espécimes sinistrais, normalmente associadas a áreas oligotróficas (Hilbrecht, 1997; Renaud \& Schmidt, 
2003). O desaparecimento do plexo G. menardii, em especial o datum fornecido pela última ocorrência da espécie G. menardii flexuosa (84 ka; Tabela 3), marca o final deste interglacial.

O aumento expressivo observado nas espécies $G$. truncatulinoides e $G$. inflata denota a chegada de águas frias e produtivas do glacial representado pela Zona Y. PortilhoRamos et al. (2006) reconheceu cinco subzonas (Y5 a Y1) no testemunho JPC-95 (bacia de Santos), com base nos horizontes fornecidos pelo plexo Pulleniatina (YP.1, YP.2 e YP.3 de Vicalvi, 1999), no entanto o biohorizonte YP.4 (limite Y1B/Y1A) não foi reconhecido pelos autores. A espécie $G$. inflata apresenta as maiores abundâncias no início do intervalo (Subzona Y2), diminuindo sua expressão média ao longo das subzonas Y1B e Y1A, enquanto G. truncatulinoides permanece constante ao longo das subzonas Y2 e Y1B, aumentando sua expressão na Subzona Y1A (Figura 6). Junto à Subzona Y2 são observados picos de abundâncias do plexo Pulleniatina e de G. crassaformis, enquanto a Subzona Y1B é caracterizada pela ausência do plexo Pulleniatina. O retorno das elevadas abundâncias do plexo $G$. menardii seguido pela brusca diminuição de G. truncatulinoides e G. inflata demonstra a chegada das águas quentes à região, marcando o início do Holoceno. As maiores abundâncias da espécie $G$. truncatulinoides observadas em testemunhos localizados mais ao sul da área de estudo (JPC-17 e JPC-95; Portilho-Ramos, 2010) sugerem ao longo do Holoceno uma maior influência de águas frias no sul da bacia, onde os valores registrados para a espécie podem ser duas vezes maiores que os observados no testemunho BS-C e BS-D.

\section{Globorotalia inflata}

O delineamento bioestratigráfico demonstrou que a espécie G. inflata apresenta uma população permanente ao longo dos últimos $\sim 620 \mathrm{ka}$ (BS-C: $\sim 620 \mathrm{ka}$; BS-D: 530 ka). Presente em intervalos glaciais e interglaciais, com abundâncias comparáveis ao plexo G. menardii em alguns intervalos (V3, V2A, V1 e X1; Figura 6), o comportamento da espécie diverge ao observado em zoneamentos realizados no Golfo do México, Mar do Caribe e Atlântico Equatorial, onde ela é a principal indicadora de águas frias sendo utilizada para o refinamento bioestratigráfico dos episódios glaciais do Pleistoceno (Bé \& Tolderlund, 1971; Kennett \& Huddlestum, 1972; Thunnel, 1984; Martins, 1990, 1993; Kohl et al., 2004). Nas bacias brasileiras, a espécie foi utilizada para a identificação de curtos períodos frios ocorridos ao longo da Zona X (Vicalvi, 1999; Sanjinés, 2006; Araújo \& Machado, 2008), onde a sua em intervalos interglaciais está associada à queda de abundância do plexo G. menardii. Este padrão não ficou claro no testemunho BS-D (Figura 4), no entanto não prejudicou a definição do zoneamento climático.

A maior representatividade da espécie ao longo dos eventos interglaciais é mais bem evidenciada quando comparados os diferentes intervalos climáticos reconhecidos nas seções (glacial e interglacial; BS-C e BS-D). Os resultados (Figura 7) sugerem que o testemunho BS-D sofreu uma maior influência de águas frias e/ou produtivas ao longo de sua história deposicional. Este fato ocorre em especial ao longo da Zona V (entre 610 e $186 \mathrm{ka}$ ), onde a espécie G. inflata (média 3,48\%) apresenta valores próximos aos observados para o plexo G. menardii (média 3,78\%) em intervalos interglaciais e comparáveis aos valores médios observados para a espécie ao longo dos intervalos glaciais do testemunho BS-C (3,50\%; Figura 7). O desenvolvimento de células de ressurgência e/ou a influência de outros fatores ambientais registrados atualmente na região, como o deslocamento da pluma do Rio da Prata e Lagoa dos Patos, que favorecida pela ação de ventos de NE propicia a chegada de águas frias, pouco salinas e muito produtivas às latitudes entre 28-23ㅇ (Campos et al., 1999; Piola et al., 2000; Gonzalez-Silveira et al., 2006), somados ao deslocamento sazonal da confluência Brasil/Malvinas podem explicar os picos de abundância da espécie $G$. inflata observados junto aos intervalos representados pelas subzonas V3, V2A, V1 e X1 (Boltovskoy et al., 2000; Portilho-Ramos et al., 2010).

\section{CONSIDERAÇÕES FINAIS}

Os intervalos bioestratigráficos definidos para o Quaternário do talude da bacia de Santos permitiram o reconhecimento das oscilações climáticas dos últimos $\sim 620 \mathrm{ka}$, e a definição de zonas e subzonas pouco conhecidas e/ou ainda não descritas para a margem continental brasileira (zonas T, U e V; subzonas V3, V2, V1, U2 e U1). A caracterização destas zonas/subzonas demonstrou que o controle do plexo Pulleniatina é uma importante ferramenta para a correlação local e possivelmente regional entre testemunhos, sendo útil não apenas para o refinamento de intervalos glaciais (Zona Y), mas também em intervalos interglaciais (Zona V). Apesar de não reconhecidas às causas de sua oscilação, o controle do plexo permitiu a proposição de novos horizontes de correlação para a bacia. Estes horizontes baseados em desaparecimentos/reaparecimentos do plexo (VP.8 a VP.1) refinaram principalmente a Subzona V3 e, junto à espécie Globorotalia menardii flexuosa, permitiram a subdivisão da Subzona V2 (duração média estimada 116 ka) em V2B (duração média 85,05 ka) e V2A(duração média 31,81 ka).

O controle do sentido de enrolamento de Globorotalia truncatulinoides, demonstrou sua aplicação bioestratigráfica, auxiliando a definição/identificação dos limites entre zonas/ subzonas. Na bacia de Santos foi útil principalmente para a definição dos limites entre as subzonas U2/U1, V2B/V2A, $\mathrm{X} 3 / \mathrm{X} 2$ e X2/X1. A distribuição vertical apresentada pela espécie Globorotalia inflata nos últimos $\sim 620 \mathrm{ka}$, demonstrou que a região sul da área de estudo apresenta uma população permanente desta espécie, sugerindo uma constante influência de águas mais frias e produtivas, no entanto, as explicações para estes fenômenos não ficaram claras neste estudo e requerem uma quantidade maior de análises.

\section{AGRADECIMENTOS}

À PETROBRAS e à UNISINOS pelo suporte ao estudo. À T.L. Dutra e R.G. Netto (ambas da UNISINOS) pelos valiosos comentários, que qualificaram o manuscrito. Aos colegas T. Wilberger e G. Krahl pelo auxílio na preparação e quarteamento das amostras. A F.A.L. Toledo e a um segundo revisor anônimo, pelos comentários que permitiram melhorar a versão final do trabalho. 


\section{REFERÊNCIAS}

Araújo, T.M.F. \& Machado, A.J. 2008. Eventos bioestratigráficos, paleoclimáticos e paleobatimétricos do talude continental superior da Bahia, evidenciados através do estudo da fauna de foraminíferos. Gravel, 6:27-45.

Bé, A.W.H.; Damuth, J.E.; Lott, L. \& Free, R. 1976. Late Quaternary Climatic Record in Western Equatorial Atlantic Sediment. In: R.M. Cline \& D.H. Hays (eds.) Investigations of Late Quaternary Paleoceanography and Paleoclimatology, p. 162200 (Geological Society of America, Memoir 145).

Bé,A.W.H. \& Tolderlund, D.S. 1971. Distribution and ecology of living planktonic foraminifera in surface waters of the Atlantic and Indian Oceans. In: B.M. Funnel \& W.R. Ridel (eds.) The micropaleontology of oceans, Cambridge University Press, p. 105-149.

Blow, W.H. 1969. Late middle Eocene to Recent planktonic foraminiferal biostratigraphy. In: INTERNATIONAL CONFERENCE ON PLANKTONIC MICROFOSSILS, 1, 1967. Proceedings, Genebra, p. 199-422.

Bolli, H.M. \& Premoli Silva, I. 1973. Oligocene to Recent planktonic foraminifera and stratigraphy of leg 15 sites in the Caribbean Sea. Initial Reports of the Deep Sea Drilling Project, 15:475-497.

Boltovskoy, E. 1968. Living planktonic foraminifera of the eastern part of the tropical Altantic. Revue de Micropaléontologie, 11:58-98.

Boltovskoy, E. 1973. Estudio de testigos submarinos del Atlántico Sudoccidental. Revista del Museo de Ciencias Naturales "Bernardino Rivadavia", 4:215-340.

Boltovskoy, E.; Boltovskoy, D. \& Brandini, F. 2000. Planktonic foraminifera from south-western Atlantic epipelagic waters: abundance, distribution and year-to-year variation. Journal of the Marine Biological Association of the United Kingdom, 79:203-213. doi:10.1017/S0025315499001794

Boltovskoy, E.; Boltovskoy, D.; Correa, N. \& Brandini, F. 1996. Planktonic foraminifera from the southwestern Atlantic $\left(30^{\circ}\right.$ $60^{\circ} \mathrm{S}$ ): species-specific patterns in the upper $50 \mathrm{~m}$. Marine Micropaleontology, 28:53-72.

Broecker, W.S.; Ewing, M. \& Heezen, B.C. 1960. Evidence for abrupt change in climate close to 11,000 years ago. American Journal of Science, 258:429-448. doi:10.2475/ajs.258.6.429

Campos, E.J.D.; Lentini, C.A.D.; Miller, J.L. \& Piola, A.R. 1999. Interannual variability of the sea surface temperature in the South Brazil Bight. Geophysical Research Letters, 26:2061-2064. doi:10.1029/1999GL900297

Chiessi, C.M.; Ulrich, S.; Mulitza, S.; Patzold, J. \& Wefer, G. 2007. Signature of the Brazil-Malvinas Confluence (Argentine Basin) in the isotopic composition of planktonic foraminifera from surface sediments. Marine Micropaleontology, 64:52-66. doi:10.1016/j.marmicro.2007.02.002

Cifelli, R. \& Beniér, C.S. 1976. Planktonic foraminifera from near the West African coast and a consideration of faunal parceling in the North Atlantic. Journal of Foraminiferal Research, 6:258273. doi:10.2113/gsjfr.6.4.258

Damuth, J.E. 1975. Quaternary climate change as revealed by calcium-carbonate fluctuations in western equatorial Atlantic sediments. Deep-Sea Research, 22:725-743. doi: 10.1016/00117471(75)90078-9

Duarte, C.S.L. \& Viana, A.R. 2007. Santos drift system: stratigraphic organization and implications for late Cenozoic paleocirculation in the Santos Basin, SW Atlantic Ocean. In: A.R. Viana \& M. Rebesco (eds.) Economic and paleoceanographic significance of contourite deposits, Society for Sedimentary Geology, p. 171-198 (Special Publication 276). doi:10.1144/GSL.SP.2007.276.01.09
Ericson, D.B. \& Wollin, G. 1956. Correlations of six cores from the Equatorial Atlantic and Caribbean. Deep-sea Research, 3:104125. doi:10.1016/0146-6313(56)90089-2

Ericson, D.B. \& Wollin, G. 1968. Pleistocene climates and chronology in deep-sea sediments. Science, 162:1227-1243. doi:10.1126/science.162.3859.1227

Ericson, D.B.; Ewing, M.; Wollin, G. \& Heezen, B.C. 1961. Atlantic deep-sea sediments cores. Geological Society of America Bulletin, 72:193-286. doi:10.1130/0016-7606(1961)72[193:AD SC]2.0.CO;2

Gibbard, P.L; Head, M.J. \& Walker, M.J.C. 2010. Formal ratification of the Quaternary System/Period and the Pleistocene Series/ Epoch with a base at $2.58 \mathrm{Ma}$. Journal of Quaternary Science, 25:96-102. doi:10.1002/jqs. 1338

González-Silveira, A.; Angel, E.S.D. \& Millán-Núñes, N. 2006. Spatial and temporal variability of the Brazil-Malvinas Confluence and the La Plata Plume as seen by Sea WIFS and AVHRR imagery. Journal of Geophysical Research, 111:C06010, 17 p. doi:10.1029/2004JC002745

Hall, I.R.; McCave, I.N.; Shackleton, N.J.; Weedon, G.P. \& Harris, S.E. 2001. Intensified deep Pacific inflow and ventilation in Pleistocene glacial times. Nature, 412:809-812. doi:10.1038/35090552

Hemleben, C.; Spindler, M. \& Anderson, O.R. 1989. Modern planktonic foraminifera. Berlin, Springer-Verlag, $363 \mathrm{p}$.

Hilbrecht, H. 1997. Morphologic gradation and ecology in Neogloboquadrina pachyderma and $N$. dutertrei (planktonic foraminifera) from core top sediments. Marine Micropaleontology, 31:31-43. doi:10.1016/S0377-8398(96)00054-0

Johns, W.E.; Lee, T.N.; Beardsley, R.C.; Candela, J.; Limeburner, R. \& Castro, B. 1998. Annual cycle and variability of the North Brazil Current. Journal of Physical Oceanography, 28:103-128. doi:10.1175/1520-0485(1998)028<0103:ACAVOT> 2.0.CO;2

Kennett, J.P. \& Huddlestun, P. 1972. Late Pleistocene paleoclimatology, foraminiferal biostratigraphy and tephrochronology, Western Gulf of Mexico. Quaternary Research, 2:38-69. doi:10.1016/0033-5894(72)90004-X

Kohl, B.; Fillon, R.H. \& Roberts, H.H. 2004. Foraminiferal biostratigraphy and paleoenvironments of the Pleistocene Lagniappe Delta and related section, Northeastern Gulf of Mexico. In: B. Anderson \& R.H. Fillon (eds.) Late Quaternary Stratigraphic Evolution of the Northern Gulf of Mexico Margin, Society for Sedimentary Geology, p. 190-216 (Special Publication 79). doi:10.2110/pec.04.79.0189

Leipnitz, I.I.; Silva, J.L.L.; Leipnitz, B.; Aguiar, E.S.; Leão, C.J.; Giovanoni, L. \& Ferreira, F. 2005. Métodos para o trabalho com microfósseis e formas atuais. In: L.L. Timm \& C.V. Cademartoti (eds.) Cadernos La Salle XI - Métodos de Estudo em Biologia, 2: 49-58.

Lidz, L. 1966. Deep-sea Pleistocene biostratigraphy. Science, 154:1448-1452. doi:10.1126/science.154.3755.1448

Lohmann, G.P. \& Schweitzer, P.N. 1990. Globorotalia truncatulinoides' growth and chemistry as probes of the past thermocline, 1, shell size. Paleoceanography, 5:55-75. doi:10.1029/PA005i001p00055

Mahiques, M.; Silveira, I.; Sousa, S.H. \& Rodrigues, M. 2002. Post-LGM sedimentation on the outer shelf of the northeastern part of the São Paulo Bight, southeastern Brazil. Marine Micropaleontology, 181:387-400.

Mahiques, M.M.; Tessler, M.G. \& Ciotti, A.M. 2004. Hydrodynamically driven patterns of recent sedimentation in the shelf and upper slope of Southeast Brazil. Continental Shelf Research, 24:1685-1697. doi:10.1016/j.csr.2004.05.013 
Martin, R.E. \& Fletcher, R.R. 1995. Graphic correlation of PlioPleistocene sequence boundaries, Gulf of Mexico: oxygen isotopes, ice volume, and sea level. Society for Sedimentary Geology, p. 235-248 (Special Publication 53).

Martin, R.E.; Johnson, G.W.; Neff, E.D. \& Krantz, D.E. 1990. Quaternary planktonic foraminiferal assemblages zones of the northeast Gulf of Mexico, Colombia Basin (Caribbean Sea), and tropical Atlantic Ocean: Graphic correlation of microfossil and oxygen isotope datums. Paleoceanography, 5: 531-555. doi:10.1029/PA005i004p00531

Martin, R.E.; Neff, E.D.; Johnson, G.W. \& Krantz, D.E. 1993. Biostratigraphic expression of Pleistocene sequence boundaries, Gulf of Mexico. Palaios, 8:155-171. doi:10.2307/3515169

Martinez, J.I; Mora, G. \& Barrows, T.T. 2007. Paleoceanographic conditions in the Western Caribbean Sea for the last $560 \mathrm{kyr}$ as inferred from planktonic foraminifera. Marine Micropaleontology, 64:177-188. doi:10.1016/j.marmicro.2007.04.004

Modica, C.J. \& Brush, E.R. 2004. Postrift sequence stratigraphy, paleogeography, and fill history of the deep-water Santos Basin, offsore southeast Brazil. AAPG Bulletin, 88:923-945. doi:10.1306/01220403043

Moreira, J.L.P.; Madeira, C.V.; Gil, J.A. \& Machado, A.P. 2007. Bacia de Santos. Boletim de Geociências da Petrobras, 15:531-549.

Neff, E.D. 1985. Pre-Late Pleistocene paleoclimatology and planktonic foraminiferal biostratigraphy of the northeastern Gulf of Mexico. Columbia University South Carolina, Dissertação de Mestrado, 123 p.

Nunes, M.C.V.; Viviers, M.C. \& Lana, C.C. 2004. Bacias sedimentares brasileiras - Bacia de Santos. Phoenix, 6:1-6.

Oliveira, D.H.; Mello, R.M.; Barreto, A.M.F. \& Strohschoen Jr., O. 2007. O limite Pleistoceno/Holoceno no Campo de Marlim Leste da Bacia de Campos - RJ, com base na bioestratigrafia de foraminíferos planctônicos. Estudos Geológicos, 17:41-50.

Patterson, R.T. \& Fishbein, E. 1989. Re-examination of the statistical methods used to determine the number of point counts needed for micropaleontological quantitative research. Journal of Paleontology, 63:245-248.

Pereira, M.J. \& Feijó, F. 1994. Bacia de Santos. Boletim de Geociências da Petrobrás, 8:219-234.

Phleger, F.B.; Parker, F.L. \& Peirson, J.F. 1953. North Atlantic Foraminifera. Reports of the Swedish Deep Sea Expedition (1947-1948), 7:1-122.

Piola, A.R.; Campos, E.J.D.; Möller Jr, O.O.; Charo, M. \& Martinez, C. 2000. Subtropical shelf front off eastern South America. Journal of Geophysical Research, 193:6565-6578. doi:10.1029/1999JC000300

Portilho-Ramos, R.C. 2010. Paleoceanografia do sudoeste do Atlântico Sul: registro de eventos abruptos nos últimos 50.000 anos. Programa de Pós-Graduação em Geoquímica Ambiental, Universidade Federal Fluminense, Tese de Doutorado, 133 p.

Portilho-Ramos, R.C.; Rios-Netto, A.M. \& Barbosa, C.F. 2006. Caracterização bioestratigráfica do Neógeno Superior da Bacia de Santos com base em foraminíferos planctônicos. Revista Brasileira de Paleontologia 9:349-354. doi:10.4072/rbp.2006.3.10

Prell, W.L. \& Damuth, J.E. 1978. The climate-related diachronous disappearance of Pulleniatina obliquiloculata in Late Quaternary sediments of the Atlantic and Caribbean. Marine Micropaleontology, 3:267-277. doi:10.1016/0377-8398(78)90031-2

Renaud, S. \& Schmidt, D.N. 2003. Habitat tracking as a response of the planktonic foraminifer Globorotalia truncatulinoides to environmental fluctuations during the last $140 \mathrm{kyr}$. Marine Micropaleontology, 49:97-122. doi:10.1016/S03778398(03)00031-8
Rodrigues, M.A.C. \& Carvalho, M.G.P. 1980. Curvas paleoclimáticas com base em foraminíferos de testemunhos da plataforma sulbrasileira. Anais da Academia Brasileira de Ciencias, 52:617-625.

Rögl, F. \& Bolli, H.M. 1973. Holocene to Pleistocene plancktonic foraminifera of leg 15, site 147 [Cariaco Basin (Trench), Caribbean Sea] and their climatic interpretation. Initial Reports of the Deep Sea Drilling Project, 15:553-616. doi:10.2973/dsdp. proc. 15.113 .1973

Sanjinés, A.E.S. 2006. Biocronoestratigrafia de foraminiferos em três testemunhos do Pleistoceno-Holoceno do talude continental da Bacia de Campos, RJ-Brasil. Programa de Pós-Graduação em Geologia, Universidade Federal do Rio de Janeiro, Dissertação de Mestrado, $119 \mathrm{p}$.

Schmidt, D.N.; Renaud, S.; Bollmann, J.; Schiebel, R. \& Thierstein, H.R. 2004. Size distribution of Holocene planktic foraminifera assemblages: biogeography, ecology and adaptation. Marine Micropaleontology, 50:319-338. doi:10.1016/S03778398(03)00098-7

Schott, W. 1935. Die foraminiferen in dem equatorialen teil des Atlantischen Ozeans. Wissenchaftliche Eregebnisse der Deutsche Atlantischen Expedition "Meteor" 1925-1927, 3:43-134.

Silveira I.C.A.; Schmidt, A.C.K.; Campos, E.J.D.; Godoi, S.S. \& Ikeda, Y. 2000. A Corrente do Brasil ao largo da costa leste brasileira. Revista Brasileira de Oceanografia, 48:171-183. doi:10.1590/S1679-87592000000200008

Thunell, R.C. 1984. Pleistocene planktonic foraminiferal biostratigraphy and paleoclimatology of the Gulf of México. In: N. Healy-Williams (ed.) Principles of Pleistocene stratigraphy applied to the Gulf of Mexico, International Human Resources Development Corporation, p. 25-64.

Toledo, F.A.L. 2000. Variações paleoceanográficas nos últimos 30.000 anos no oeste do Atlântico Sul: isótopos de oxigênio, assembléias de foraminiferos planctônicos e nanofósseis calcários. Programa de Pós-Graduação em Geociências, Universidade do Rio Grande do Sul, Tese de Doutorado, 245 p.

Toledo, F.A.L.; Costa, K.B. \& Pivel, M.A. 2007. Salinity changes in the western tropical South Atlantic during the last $30 \mathrm{kyr}$. Global and Planetary Change, 57:383-395. doi:10.1016/j. gloplacha.2007.01.001

Viana, A.R.; Faugères, J.C; Kowamann, J.A.M.; Lima, J.A.M.; Caddah, L.F.G. \& Rizzo, J.G. 1998. Hydrology, morphology and sedimentology of the Campos continental margin, offshore Brazil. Sedimenatary Geology, 115:133-157. doi:10.1016/ S0037-0738(97)00090-0

Vicalvi, M.A. 1997. Zoneamento bioestratigráfico e paleoclimático dos sedimentos do Quaternário superior do talude da Bacia de Campos, RJ, Brasil. Boletim de Geociências da Petrobrás, 11:132-165.

Vicalvi, M.A. 1999. Zoneamento bioestratigráfico e paleoclimático do Quaternário Superior do talude da Bacia de Campos e Platô de São Paulo adjacente, com base em foraminíferos planctônicos. Programa de Pós-graduação em Geologia, Universidade Federal do Rio de Janeiro, Tese de Doutorado, 183 p.

Vicalvi, M.A. \& Palma, J.J.C. 1980. Bioestratigrafia e taxas de acumulação dos sedimentos quaternários do talude do sopé continental entre a Foz do Rio Gurupi (MA) e Fortaleza (CE). Boletim Técnico da Petrobrás, 23:3-11.

Wainer, I. \& Venegas, S.A. 2002. South Atlantic multidecadal variability in the climate system model. Journal of Climate, 15:1408-1420. doi:10.1175/1520-0442(2002)015<1408:SAM VIT>2.0.CO;2

Received in November, 2011; aceepted in June, 2012. 